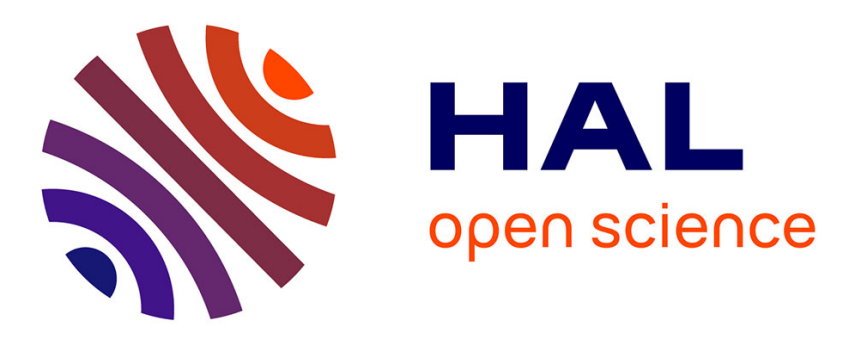

\title{
Global transition dynamics of flow in a lid-driven cubical cavity
}

Rajesh Ranjan, Sasidharan Unnikrishnan, Jean-Christophe Robinet, Datta

Gaitonde

\section{- To cite this version:}

Rajesh Ranjan, Sasidharan Unnikrishnan, Jean-Christophe Robinet, Datta Gaitonde. Global transition dynamics of flow in a lid-driven cubical cavity. Theoretical and Computational Fluid Dynamics, 2021, 35 (3), pp.397-418. 10.1007/s00162-021-00565-z . hal-03277000

\section{HAL Id: hal-03277000 https://hal.science/hal-03277000}

Submitted on 2 Jul 2021

HAL is a multi-disciplinary open access archive for the deposit and dissemination of scientific research documents, whether they are published or not. The documents may come from teaching and research institutions in France or abroad, or from public or private research centers.
L'archive ouverte pluridisciplinaire HAL, est destinée au dépôt et à la diffusion de documents scientifiques de niveau recherche, publiés ou non, émanant des établissements d'enseignement et de recherche français ou étrangers, des laboratoires publics ou privés. 


\title{
Global transition dynamics of flow in a lid-driven cubical cavity
}

\begin{abstract}
The dynamics of a fully three-dimensional lid-driven cubical cavity (3D-LDC) flow at several postcritical conditions, i.e., beyond the first bifurcation, are elucidated using both linear and nonlinear analyses. When the Reynolds number is increased beyond the critical value, symmetry breaks down intermittently with subsequent gradual growth in spanwise inhomogeneity. This results in crossflow as well as pronounced secondary flow due to enhanced imbalance between centrifugal and pressure forces. Thus, while a stable solution is obtained at $\operatorname{Re}=1900$ (Reynolds number based on lid velocity and cavity side length), nonlinear analysis identifies intermittent and nearly saturated regimes at $\operatorname{Re}=2100$ and $\operatorname{Re}=3000$, respectively. These changes in the regime are examined by considering five basic states at different Reynolds numbers starting from $\operatorname{Re}=1900$. At the lowest Reynolds number, linear analysis yields only symmetric modes, characterized by Taylor-Görtler-like (TGL) vortices. However, at $\mathrm{Re}=2100$, the intermittent breakdown of symmetry results in the emergence of an antisymmetric low-frequency mode apart from primary high-frequency mode. The frequencies of both these modes are numerically close to those obtained from corresponding nonlinear simulations. When the Reynolds number is increased even further, the TGL structures drift under the influence of the crossflow to occupy the previously structureless region near the wall. The frequency of each mode decreases with increase in Re; between 1900 and 3000, the frequency of the primary mode changes by more than $20 \%$. Furthermore, the spatial support of each mode becomes larger within the cavity. Both primary and secondary modes are increasingly destabilized with Re; however, the secondary antisymmetric mode is destabilized at a higher rate. The current study thus provides a comprehensive picture of the overall dynamics of 3D-LDC flows in pre- and post-bifurcation regimes in an extended Re range not considered hitherto.
\end{abstract}

Keywords TGL vortices $\cdot$ Linear stability $\cdot$ Global modes

Communicated by Sergio Pirozzoli.

This material is based partly upon work supported by the Air Force Office of Scientific Research under Award Number FA955017-1-0228 (monitor: Gregg Abate).

R. Ranjan $(\varangle) \cdot$ D. Gaitonde

Mechanical and Aerospace Engineering, The Ohio State University, Columbus, OH 43210, USA

E-mail: rajeshr@iitk.ac.in

R.Ranjan

Present address:

Department of Aerospace Engineering, Indian Institute of Technology, Kanpur 208016, India

S. Unnikrishnan

Department of Mechanical Engineering, Florida State University, Tallahassee, FL 32310, USA

J.-C. Robinet

DynFluid Lab., Arts \& Métiers Paris - 151, Bd. de l’Hôpital, 75013 Paris, France 


\section{Introduction}

Flow in a lid-driven cavity (LDC) is a classical benchmark problem in fluid dynamics, frequently employed to assess robustness and accuracy of numerical schemes because of its simple geometrical configuration and welldefined boundary conditions. Its rich dynamics are also representative of several other flowfields encountered in nature as well as in engineering applications. Therefore, a more fundamental application of the LDC problem is to explore successive flow bifurcations from subcritical to supercritical conditions, which facilitates a better understanding of important instability mechanisms as well as the role of nonlinearity with increase in Reynolds number.

The two-dimensional lid-driven cavity (2D-LDC) is arguably one of the most examined flows in the context of stability dynamics; a detailed review is presented in Erturk [9]. The four walls in this flow are referred to as top, bottom, upstream and downstream, respectively, as shown in Fig. 1a. The flow is characterized by a central primary eddy (PE) and surrounding secondary eddies, designated as DSE (downstream secondary eddy), USE (upstream secondary eddy) and TSE (top secondary eddy). The momentum transfer from the top moving lid creates a downward wall jet on the top corner of the downstream wall. The jet later separates from this wall and impinges on the bottom wall to form the DSE. The wall jet then separates from the bottom wall at some distance from the left corner and impinges on the upstream sidewall resulting in the USE. Depending on the Reynolds number, separation may occur again near the top left corner. The central primary eddy consists of relatively slower moving rotating fluid, and the transfer of momentum from the wall jet into this core depends on the Reynolds number [42].

The stability dynamics of fully three-dimensional lid-driven cavity (3D-LDC) flows are more pertinent to realistic applications. However, their analysis is computationally very expensive, and comprehensive efforts have appeared only more recently. The literature suggests that the dynamics of 3D-LDC flows differ significantly from their 2D counterparts [44]. The 3D-LDC configuration, as shown in Fig. 1b, contains two end walls in spanwise $(z-)$ direction; the central $x y$-plane in between is denoted the symmetry plane. The prominent flow features of 2D-LDC are observed on this plane at low Reynolds numbers [14]. As the Reynolds number is increased, however, the presence of the third dimension affects the flow in ways that distinguish the 3D-LDC from its 2D-LDC counterpart. Specifically, prominent features, known as Taylor-Görtler-like (TGL) vortices, appear on the side walls as displayed in Fig. 1b. Corner vortices may also form at the cavity end walls (not shown). The spanwise inhomogeneity results in crossflow as the Reynolds number is increased. An early experimental effort [23] examining wall effects found that corner vortices influence the downstream secondary eddy (DSE), whose size diminishes with Reynolds number when it is greater than 2000.

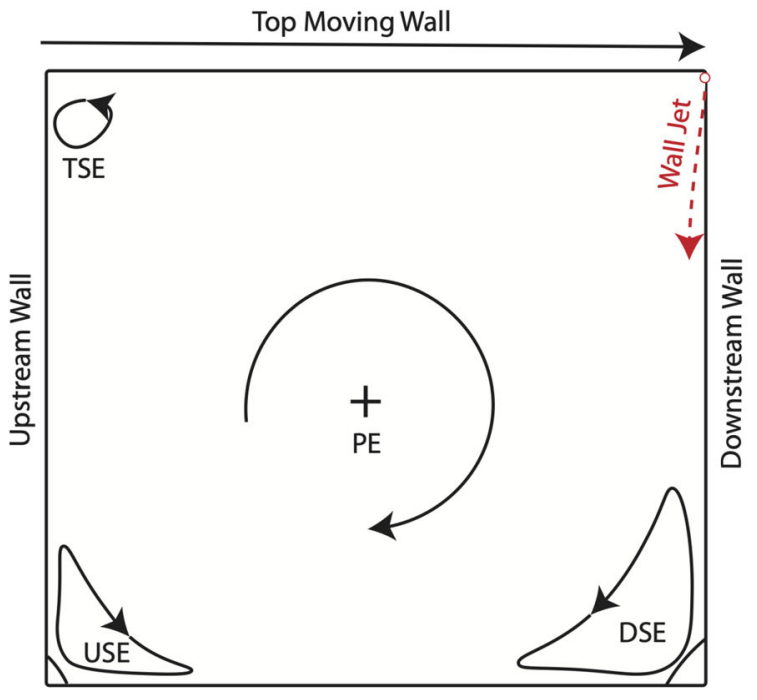

(a) $2 \mathrm{D}$

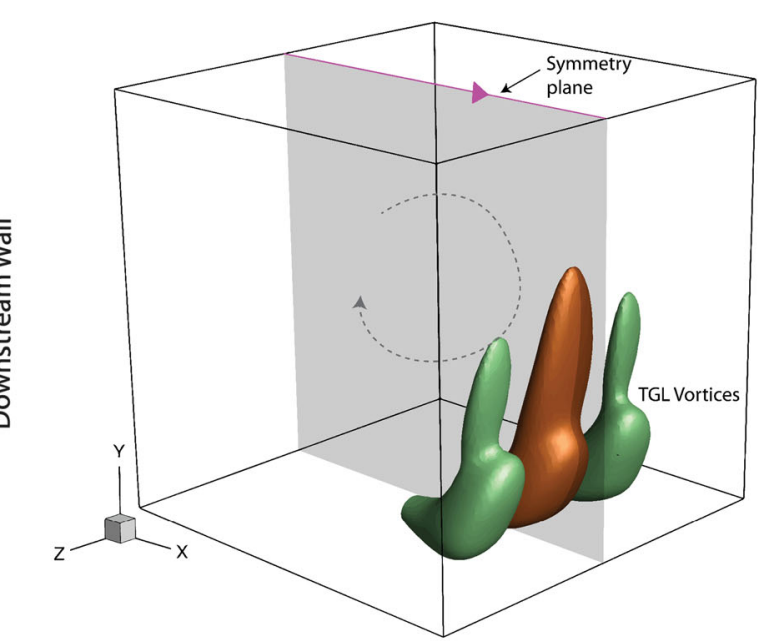

(b) $3 \mathrm{D}$

Fig. 1 Flow topology for LDC flows. PE Primary eddy, DSE downstream secondary eddy, USE upstream secondary eddy, TSE top secondary eddy, TGL Vortex Taylor-Görtler-like vortex. Sudden turning of flow at the top corner of the downstream sidewall creates a downward oriented flow that behaves like a variable-thickness wall jet shown in (a) 
Table 1 Stability studies in the literature for the lid-driven cubical cavity (LSA linear stability analysis, TI time integration)

\begin{tabular}{lllll}
\hline References & Approach & $\pm \omega_{\text {cr }}$ & $\operatorname{Re}_{\text {cr }}$ & $\operatorname{Re}>\operatorname{Re}_{\text {cr }}$ \\
\hline Giannetti et al. [16] & LSA & 0.0 & $\gtrsim 2000$ & 2100 \\
Feldman and Gelfgat [11] & TI & 0.575 & 1914 & $1925,1945,1970$ \\
Gómez et al. [17] & LSA & 0.5718 & $<2000$ & - \\
Kuhlmann and Albensoeder [25] & TI & 0.586 & 1919.5 & 1921,1970 \\
Anupindi et al. [2] & LES & 0.012 & $>2100$ & $2250,2300,2350$ \\
Loiseau et al. [29] & LSA & 0.585 & 1914 & 1930,1970 \\
Lopez et al. [30] & TI & 0.5832 & 1928.9 & {$[19302100]$} \\
Gelfgat [15] & LSA & 0.5861 & 1919.4 & - \\
\hline
\end{tabular}

The earliest attempts at performing stability analysis in 3D-LDC used a two-dimensional base flow with a Fourier wave assumption in the spanwise direction [1,49]; this essentially emulates an LDC flow with periodic boundaries at a specified wavenumber. The bifurcation in such flows has been shown to be supercritical [1], and TGL structures appear, which constitute a primary mechanism of instability. The extent to which the stability of the flow is affected thus depends on the spanwise length of the cavity as well as the Reynolds number, Re. Theofilis et al. [50] report that the inclusion of the periodic spanwise direction in the flow brings the critical Reynolds number to $\mathrm{Re}_{\mathrm{cr}} \simeq 782$, which is an order of magnitude lower than the critical value of the pure $2 \mathrm{D}$ counterpart $\left(\operatorname{Re}_{\mathrm{cr}}^{2 D} \simeq 8000\right)$. However, the viscous effects due to the presence of sidewalls, such as damping of TGL structures, cannot be studied in this periodic spanwise configuration.

A primary limiting factor in performing fully three-dimensional stability analyses with end-wall effects is the difficulty in solving the large eigenvalue problem that arises naturally in the formulation of the problem. The evolution of iterative eigenvalue solvers such as those based on the Arnoldi iteration method [48] has alleviated this problem and has recently facilitated 3D-LDC studies. High-fidelity discretization schemes [38], as well as the availability of computational power, have also aided the analysis. Some investigators, as reported below, have approached the stability problem through direct time integration (TI) of high-resolution computational data, which demands less computational memory but more processor resources. While the eigenvalue approach yields the frequencies and growth rates, as well as structures of global modes, the TI approach is often useful in obtaining the precise bifurcation point, together with the nature of transition (such as subcritical or supercritical).

Table 1 lists several major recent advances in the stability dynamics of fully three-dimensional LDC flows, along with their numerical approaches, in chronological order (cf. Kuhlmann and Romanò [26]; Feldman [10] for cavities with different aspect ratios as well as configurations). Apart from the critical parameters at bifurcation, the list also includes the post-bifurcation Reynolds numbers $\left(\operatorname{Re}>\operatorname{Re}_{\mathrm{cr}}\right)$ examined, as this aspect is central to the current study.

Giannetti et al. [16] were the first to examine the 3D-LDC cavity with the traditional stability approach using the Arnoldi method, but on a relatively modest $\left(64^{3}\right)$ mesh. They found that the flow is stable at $\operatorname{Re}=2000$ but becomes unstable at 2100. Numerical convergence issues such as those due to low resolution were not addressed. Subsequently, both Feldman and Gelfgat [11] and Kuhlmann and Albensoeder [25] used timeintegration methods with rigorous convergence studies and found similar critical parameters at bifurcations. The transition from steady to oscillatory flow was confirmed to be weakly subcritical in these studies.

Analyses of fully three-dimensional LDCs on reasonably refined grids using the traditional linear stability approach with eigenvalue problem formulation have become available more recently. Gómez et al. [17]; Loiseau et al. [29] used this approach and extracted frequencies similar to those obtained with the TI approach in Table 1 and displayed the structure of this mode as being characterized by symmetric TGL structures about the midplane. Loiseau et al. [29] also reported the critical Reynolds number as $\operatorname{Re}_{\mathrm{cr}} \simeq 1914$, which was further confirmed by Gelfgat [15] from stability analysis on a very fine grid $\left(256^{3}\right)$. Anupindi et al. [2] used LatticeBoltzmann simulations to observe steady flow at $\mathrm{Re}=2100$. The flow became oscillatory only at $\mathrm{Re}=2250$ and also exhibits a very different frequency than reported by others. This discrepancy may be attributable to the low grid resolution $\left(80^{3}\right)$ or, as identified by Gelfgat [15], to the change in momentum equation with the eddy model used.

The above discussion makes it evident that there is generally a broad agreement in the literature about the critical parameters for instability of cubical cavity flows at the first Hopf bifurcation point and the subcritical nature of transition. Some recent studies, shown in the last column in Table 1, also describe flow evolution beyond $\mathrm{Re}_{\mathrm{cr}}$ such as presence of secondary bifurcations, limit cycles and associated changes of the symmetry of 
flow. Both Feldman and Gelfgat [11] and Kuhlmann and Albensoeder [25] have considered slightly supercritical flows in their studies, but their observations differ on the effects of nonlinearity. Two key events in the immediate aftermath of bifurcation are: the breakdown of symmetry and the appearance of intermittent bursts. Feldman and Gelfgat [11] noted loss of symmetry soon after bifurcation and regular oscillations with saturated amplitude for all $\mathrm{Re}>\mathrm{Re}_{\mathrm{cr}} \simeq 1914$. However, Kuhlmann and Albensoeder [25] observed loss of symmetry only at $\operatorname{Re}=1970$, while at slightly supercritical conditions $\left(\operatorname{Re}_{\mathrm{cr}}<\operatorname{Re} \leq 1970\right)$, the dynamics are characterized by intermittent events. These intermittent bursts were further confirmed in the simulations of Loiseau et al. [29], who observed two limit cycles corresponding to primary oscillations and intermittent events, respectively. The frequencies of these two cycles differ by a ratio of about 4. Lopez et al. [30] presented a comprehensive analysis of transition to oscillatory dynamics by gradually increasing the Reynolds number limit to 2100 . They attributed the breakup of reflection symmetry about the midplane as observed in nonlinear simulations to a second Hopf bifurcation at $\mathrm{Re}=2089$. The eigenfrequency of this bifurcation is close to that of the intermittent large-amplitude oscillations as observed by Loiseau et al [29]. A more detailed discussion of these observations is provided in a recent review by Kuhlmann and Romanò [26].

Several experimental $[23,27]$ and numerical $[2,20]$ studies report significant changes in the character of the flow in the 2000 to 3000 Reynolds number range, particularly on the intensity and distribution of the TGL vortices. Shankar and Deshpande [44] in their review suggest that the number of TGL vortex pairs increases with Re, influencing the spanwise motion and significantly altering the underlying flow dynamics. However, rigorous stability studies examining Re effects in this limit are sparse and most studies in Table 1 have been confined in the immediate vicinity of $\mathrm{Re}_{\mathrm{cr}}$, i.e., within $8 \%$ of $\mathrm{Re}_{\mathrm{cr}} \simeq 1914$. Since the $\mathrm{Re}$ span over which the flow changes from stable to oscillatory is relatively small in these works, no appreciable changes in the frequencies of dominant modes or their structures (as expected due to change in side-wall viscous drag) are reported. An exception in Table 1 is the study by Anupindi et al. [2], who extended the analysis for a slightly higher Re range up to 2350 . An interesting observation was that the flow loses symmetry at $R e=2250$, but it gets restored at $\mathrm{Re}=2350$. As noted earlier, although the eddy model used in the study may have affected the prediction of critical parameters, the observation is nonetheless interesting in its own right, meriting further examination. Overall, the understanding of the evolution of the flow dynamics in 3D-LDC flow in the Reynolds number range $2000<\operatorname{Re}<3000$ is generally limited. The purpose of the present study is to examine the change in flow behavior in this extended Re-range using both linear and nonlinear simulations and analysis. Particularly, the study focuses on tracking the change in the dominant frequency and therefore the structure of the mode, as the flow parameters are changed away from the critical values.

The approach in the present study differs from those described earlier, which investigated the postbifurcation mechanism in detail. In the Re-range considered here $(1900 \leq \operatorname{Re} \leq 3000)$, the flow goes through transition from stable to oscillatory to chaotic intermittent to almost saturated state. Therefore, following the successive bifurcations and computing their stability as the flow transitions to turbulence become quickly impossible or even a totally illusory task. In fact, computing the basic states and their stabilities becomes very challenging as soon as the solution is no longer quasi-periodic [5]. On the contrary, when the flow is fully turbulent, two approaches are currently possible. The first consists in computing the stabilities of particular solutions like the exact coherent states (ECS; Park and Graham [39]), and the turbulent solution is then seen as a particular trajectory (in phase space) around these ECS. The notion of the turbulent solution as a weighted combination of ECS, although attractive, is strongly dependent on the initial conditions and is therefore not robust or very predictive. The other solution is to adopt a more statistical description which, to first order, is represented by a very long time-averaged state. This state describes an asymptotic solution of all trajectories in phase space.

The time-averaged flow unlike the steady basic flow, which is a fixed point of the Navier-Stokes equations, is not a solution of the latter but can be perceived as that of the Reynolds-averaged equations. In addition, the timeaveraged flow differs from the steady basic flow by a distortion term which is due to nonlinear interactions [34, 47]. This peculiarity is all the more true as the flow regime is far from the threshold of appearance of the bifurcation. When weakly nonlinear theory becomes invalid for a flow state beyond bifurcation, it has been argued that the mean flow can be used to redefine the concept of an equilibrium point $[34,47]$ and sometimes can constitute a more suitable choice than the steady base flow to characterize the modes [5]. For example, Barkley [3] found that in cylinder wakes over a range of Re, the frequency of the vortex shedding can be tracked through stability analysis of the mean flow. Later, Mittal [35] also observed similar behavior, i.e., stability analysis of the mean flow accurately predicted the vortex-shedding frequency; however, results obtained from the steady base flow severely under-predicted it. Similar examples of successful frequency prediction using linearization around the mean flow are available for transitional [36] and turbulent round jets [18], flat plates [33] and airfoils [8]. 
Mean flows in these examples are obtained either by directly solving Reynolds-averaged equations or by timeaveraging instantaneous flowfields from large eddy simulation (LES) or direct numerical simulation (DNS). Both approaches are suitable for obtaining essential dynamics as shown in Mettot et al. [34]. An additional support for mean flow approach in the current study is due to the confined configuration of the LDC flow that dictates the leading dynamics in the flow. Bengana et al. [5] have observed that for flows within confined walls, frequencies and growth rates predicted by a linear analysis around the mean flow are closer to the truth model (DNS) than those obtained with the base flow. Overall, the geometrical constraints place the LDC in the category of flows with a dominant singular value; in this case, the spatial structure of the unsteadiness at unstable frequencies may be closely related to the dominant singular mode around the mean flow. Mean flow stability analysis then suggests a way to accurately predict unsteady features of a flow, as successfully shown in the case of backward facing step [4] and open cavity [5].

Based on the above discussion, the present work adopts mean flow as the equilibrium point to extract the linear dynamics of flows at different Reynolds numbers, while the results are also compared with fully nonlinear simulations. This mean flow is obtained by time-averaging the snapshots as obtained by LES with the approach described in Sect. 2(a). A gradual change in the flow dynamics for the lid-driven cavity around the equilibrium point is studied by considering five Reynolds numbers in $1900 \leq \operatorname{Re} \leq 3000$. The methodology for linear evolution of the flow is presented in Sect. 2(b). Sections 3(a) and (b) delineate results of pre- and post-bifurcation dynamics, respectively. A phenomenological description of the mean flow at different states is first provided. The evolution of viscous and nonlinear effects with Re is then characterized through dominant mode structures and their associated frequencies. Finally, some concluding remarks are made in Sect. 4.

\section{Methodology}

\subsection{Nonlinear evolution}

The compressible Navier-Stokes equations are solved in non-dimensional form on a curvilinear $(\xi, \eta, \zeta)$ coordinate system:

$$
\frac{\partial}{\partial \tau}\left(\frac{Q}{J}\right)=-\left[\left(\frac{\partial F_{i}}{\partial \xi}+\frac{\partial G_{i}}{\partial \eta}+\frac{\partial H_{i}}{\partial \zeta}\right)+\frac{1}{R e}\left(\frac{\partial F_{v}}{\partial \xi}+\frac{\partial G_{v}}{\partial \eta}+\frac{\partial H_{v}}{\partial \zeta}\right)\right]
$$

where $Q=[\rho, \rho u, \rho v, \rho w, \rho E]^{T}$ is the solution vector, defined in terms of the fluid density $\rho$, Cartesian velocity components $(u, v, w)$ and total specific internal energy $E=T /(\gamma-1) M^{2}+\left(u^{2}+v^{2}+w^{2}\right) / 2$. Here, $M$ is the reference Mach number of the flow, $\gamma$ is the ratio of the specific heats and $T$ is the fluid temperature. The ideal gas law connects fluid pressure $p$ to $\rho$ and $T$ as $p=\rho T / \gamma M^{2}$. Sutherland's law is used to express fluid viscosity $\mu$ as a function of temperature $T . J=\partial(\xi, \eta, \zeta, \tau) / \partial(x, y, z, t)$ denotes the Jacobian of the transformation from Cartesian $(x, y, z)$ to curvilinear $(\xi, \eta, \zeta)$-coordinate system. The inviscid and viscous fluxes in $(\xi, \eta, \zeta)$-directions are represented in Eq. (1) by $\left(F_{i}, G_{i}, H_{i}\right)$ and $\left(F_{v}, G_{v}, H_{v}\right)$, respectively. The expression for the inviscid flux $F_{i}$ is given as

$$
F_{i}=\left[\begin{array}{c}
\rho U \\
\rho u U+\widehat{\xi}_{x} p \\
\rho v U+\widehat{\xi}_{y} p \\
\rho w U+\widehat{\xi}_{z} p \\
(\rho E+p) U-\widehat{\xi}_{t} p
\end{array}\right]
$$

where $U=\left(\widehat{\xi}_{t}+\widehat{\xi}_{x} u+\widehat{\xi}_{y} v+\widehat{\xi}_{z} w\right), \widehat{\xi}_{x}=J^{-1} \partial \xi / \partial x, \widehat{\xi}_{y}=J^{-1} \partial \xi / \partial y$ and $\widehat{\xi}_{z}=J^{-1} \partial \xi / \partial z$. The expressions for other two inviscid flux quantities $G_{i}$ and $H_{i}$ can also be similarly defined by replacing $\xi$ in Eq. (2) by $\eta$ and $\zeta$, respectively. The viscous flux $F_{v}$ is given as

$$
F_{v}=\left[\begin{array}{c}
0 \\
\widehat{\xi}_{x_{i}} \tau_{i 1} \\
\widehat{\xi}_{x_{i}} \tau_{i 2} \\
\widehat{\xi}_{x_{i}} \tau_{i 3} \\
\widehat{\xi}_{x_{i}}\left(u_{j} \tau_{i j}+\frac{1}{(\gamma-1) M^{2}} \frac{1}{P r} q_{i}\right)
\end{array}\right]
$$


where Pr denotes the Prandtl number of the fluid. The stress tensor $\tau_{i j}$ and heat flux $q_{i}$ appearing in Eq. (3) are given as

$$
\begin{aligned}
\tau_{i j} & =\mu\left(\frac{\partial \xi_{k}}{\partial x_{j}} \frac{\partial u_{i}}{\partial \xi_{k}}+\frac{\partial \xi_{k}}{\partial x_{i}} \frac{\partial u_{j}}{\partial \xi_{k}}-\frac{2}{3} \delta_{i j} \frac{\partial \xi_{l}}{\partial x_{k}} \frac{\partial u_{k}}{\partial \xi_{l}}\right) \\
q_{i} & =\mu \frac{\partial \xi_{j}}{\partial x_{i}} \frac{\partial T}{\partial \xi_{j}}
\end{aligned}
$$

Expressions for other two viscous fluxes, namely $G_{v}$ and $H_{v}$, can also be similarly defined by replacing $\xi$ in Eq. (3) by $\eta$ and $\zeta$, respectively.

The discretized equations are solved using high-order schemes as described in Gaitonde and Visbal [13]. Specifically, a sixth-order compact difference scheme is used for the fluxes, together with an eighth-order implicit filter to inhibit numerical issues associated with nonlinearities and boundary conditions. A secondorder implicit scheme is used for time-marching to wash out initial transients in the flow. Subsequently, a nonlinearly stable third-order Runge-Kutta method [45] is employed to obtain either a steady flow or a statistically stationary asymptote, depending on Re. For the latter case, a long time average is computed, during which time snapshots are also stored as necessary. No-slip boundary conditions are used on all cavity walls. All velocity components are set to zero on the boundaries, except for the top wall, where the lid velocity is assigned to the $u$-component. Neumann boundary conditions are used for pressure and density.

\subsection{Linear evolution}

The linear evolution of perturbations around an equilibrium point displays their growth or decay with time, typically associated with large-scale structures [34]. These perturbations snapshots are often obtained using a linearized Navier-Stokes equations (LNSE) solver or a regular Navier-Stokes solver combined with Frèchet derivatives [17, 19,31]. In the present work, we use a high-order Navier-Stokes-mean flow perturbation (NSMFP) approach, described in Ranjan et al. [41], which uses a numerical linearization [51] to facilitate the use of the same code (Sect. 2.1) that solves nonlinear equations. The procedure is illustrated in Fig. 2 and briefly described below. The Navier-Stokes equations in compact notation can be written as,

$$
\frac{\partial \boldsymbol{q}}{\partial t}=\mathscr{N}(\boldsymbol{q})
$$

where $\mathscr{N}$ is the nonlinear Navier-Stokes operator. A steady base flow satisfies the above equations exactly. However, the mean flow, depending on the state of flow (below or above bifurcation point), may or may not satisfy above equations. Therefore, in the NS-MFP approach, the change in the mean flow, $\Delta \overline{\boldsymbol{q}}$, due to the Navier-Stokes operator is obtained by solving equation (6) for one time step and then stored for subsequent usage. For the evolution of perturbations, the Navier-Stokes equations are first marched in time for both the mean flow and the added initial perturbation, $\overline{\boldsymbol{q}}+\boldsymbol{q}^{\prime 0}$, as shown by the thick black arrow in Fig. 2 . This updates both the mean flow and perturbation, but the evolution of mean flow due to numerical or physical reasons is arrested by subtracting $\Delta \overline{\boldsymbol{q}}$, obtained earlier, as shown by the green dashed downward arrow in the figure. Thus, at this state, indicated by red dots in the figure, the mean flow remains unaltered, but the linear evolution of only the perturbation snapshots $\boldsymbol{q}^{\prime}$, under the assumption of small initial perturbation, is obtained. This procedure is repeated every time step to obtain evolution with time as shown in the figure, while the perturbation snapshots are stored for post-processing by subtracting the mean flow, $\overline{\vec{q}}$ (orange downward arrow).

Linear perturbation simulations are performed with the same flux schemes as the nonlinear ones, and time-stepping is performed using the explicit third-order Runge-Kutta method. NS-MFP approach offers flexibility in applying boundary conditions to either only on perturbations $\boldsymbol{q}^{\prime}$ or total flow variables $\overline{\boldsymbol{q}}+\boldsymbol{q}^{\prime}$. In the current work, the boundary conditions are applied to the total flow variables, and thus, Dirichlet and Neumann boundary conditions are applied, respectively, on velocity and pressure (and density) in the manner used in nonlinear simulations. Although the current study considers essentially incompressible conditions, the numerical approach of linear and nonlinear evolution can analyze compressibility effects on stability with increase in Mach number. The resulting stabilizing influence has been discussed for two-dimensional lid-driven cavities [37] and cylinders [7]. 


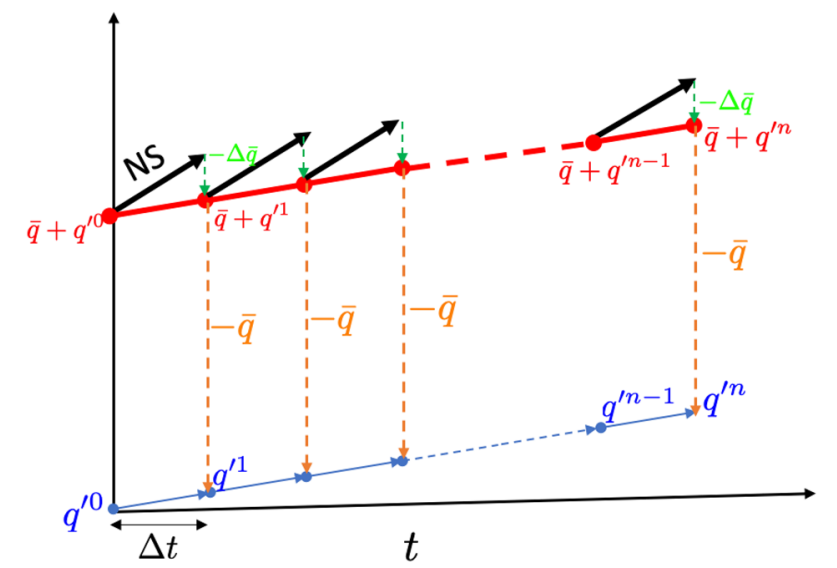

Fig. 2 Linear evolution of initial disturbance field $\boldsymbol{q}^{\prime 0}$ superimposed on mean flow $\overline{\boldsymbol{q}}$ using the NS-MFP approach. The thick black arrow shows evolution of both the mean flow and perturbations using Navier-Stokes operator. The changes to the mean flow are eliminated at each time step by subtracting $\Delta \bar{q}$, shown by green downward arrow; this ensures that only the perturbation is evolved (color figure online)

\section{Results and discussion}

The lid-driven cubical cavity considered is similar to that used in the literature as shown earlier in Fig. 1b. The computational domain is comprised of a cube in $\left[\begin{array}{ll}0 & 1\end{array}\right]^{3}$ with 121 grid points uniformly distributed in each direction; a grid convergence study of the mean flow, performed with a $201^{3}$ grid as presented later, indicates no significant change in the flow. The top lid at $y=1$ moves in positive $x$ direction, whereas $z$ represents the spanwise direction. The symmetry $x y$-plane thus lies at $z=0.5$. The length and velocity scales chosen for non-dimensionalization are the cavity side length, $L$ and top lid velocity $U$, respectively.

The simulations are initialized with quiescent flow in the cavity. The initial transients typically require about 100 flow characteristic times, $T_{c} \equiv \frac{t U}{L}$, during which the meaningful physical structures develop. A suitable mean flow for the subsequent linear analysis is obtained by computing the average over a minimum of 200 flow characteristic times after discarding the initial transients. The time-step size used to generate linearized snapshots with the third-order Runge-Kutta scheme is $\Delta t=2 \times 10^{-4}$. These snapshots are sampled at every 200 iterations, and thus, the effective time step for the subsequent stability analysis is 0.04 . The highest resolvable Strouhal number $S t=12.5$ (circular frequency $\omega \simeq 78$ ) using the Nyquist criterion is much higher than any frequency of interest. Wherever possible, the mean flow is compared to those available in the literature before stability analysis.

The results are presented in two parts. In the first, the flow is examined with numerical simulations and stability analyses just prior to bifurcation. Validation of the current method to extract stability characteristics is also provided. The second part focuses on flow dynamics after the transition to oscillatory flow. Five Reynolds numbers, 1900, 2100, 2400, 2700 and 3000, are considered, to encompass the phenomena of interest, including the evolution of global modes and dominant frequencies.

\subsection{Dynamics near bifurcation}

The dynamics near bifurcation are examined at $\mathrm{Re}=1900$, where a stable solution is obtained as also expected from prior studies at this flow parameter (see Table 1). The features of the basic state, as obtained by timeaveraging, are shown in Fig. 3a, b with surface-restricted streamlines at the symmetry plane as well as at a plane close to the end wall $(z=0.05)$. As expected, the streamlines at the symmetry plane resemble the flow in a 2DLDC (see Fig. 1a), with the presence of a primary central eddy, and also upstream and downstream secondary eddies. The top secondary eddy as found in the 2D-LDC flow at its critical conditions, $\operatorname{Re}_{\mathrm{cr}}^{2 D} \simeq 8000$ [41], is absent in the 3D-LDC because of the smaller Re. The streamlines in this plane do not indicate any effect of the end walls at the midplane suggesting the absence of any spanwise flow component. However, end-wall effects are evident in the streamlines on planes near the walls (Fig. 3b), where the inward spiraling motion of the fluid particles is clearly visible. The open streamlines indicate the presence of secondary flow, which arises due to 


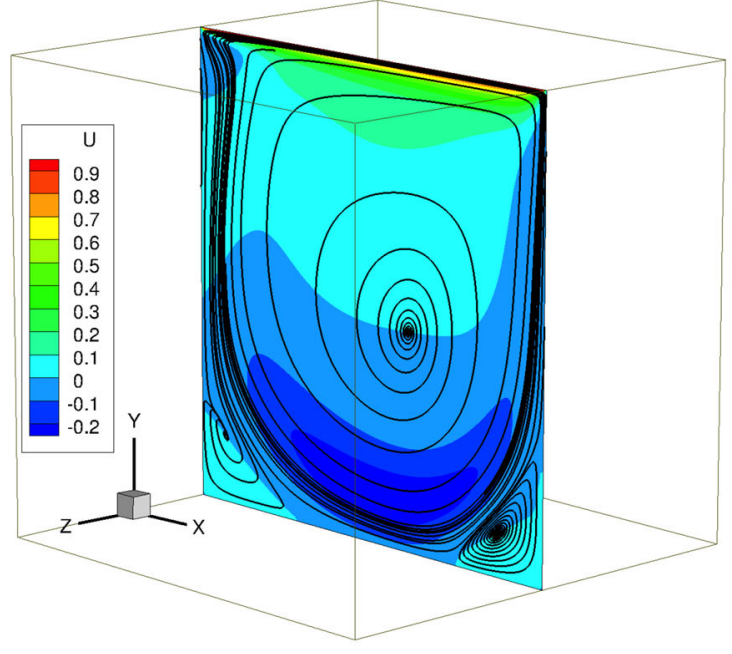

(a) Symmetry plane $(z=0.5)$

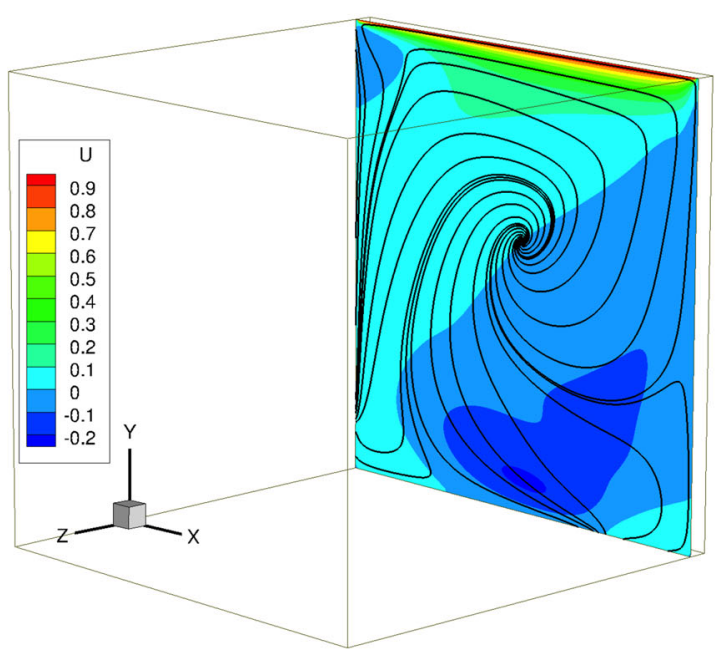

(b) Near end wall $(z=0.05)$

Fig. 3 Streamlines of the basic state. Contours of streamwise velocity are also shown. The wall at the top moves in the $+v e$ $x$-direction, $\operatorname{Re}=1900$

the imbalance between pressure and centrifugal forces. The transverse velocity component $w$ at this plane is nonzero, but decays exponentially from the wall and vanishes at the midplane as shown in Fig. 3a.

Quantitative validation of the basic state at the symmetry plane is shown in Fig. 4a by comparing with the results of Feldman and Gelfgat [11], who used a Richardson extrapolation based on $152^{3}$ and $200^{3}$ grids to obtain steady-state results. The figure shows good agreement between these two datasets. Both $u$ and $v$ velocities vary rapidly near the walls because of the no-slip effects of side walls. Figure $4 \mathrm{~b}$ shows the variation of transverse velocity component $w$ along the spanwise direction $z$ at three locations: at the center, as well as in the vicinity of secondary eddies near the bottom of upstream and downstream walls. The $w$ component is antisymmetric with respect to the symmetry plane. Its value is zero at this plane at all the three locations, further confirming no crossflow at the center of the cavity, as earlier observed from streamlines in Fig. 3.

Linear analysis is performed around this basic state. The linearized snapshots are first obtained using the NSMFP approach given in Sect. 2.2. An initial impulse random perturbation field is specified to the pressure with a magnitude of $\epsilon=10^{-5} P_{\text {ref }}$, where $P_{\text {ref }}$ is the reference pressure. Snapshots were also processed by doubling the perturbation magnitude; the results changed proportionally, thus confirming linearity. The snapshots effectively constitute a subspace similar to Krylov subspace with an initial random vector in Arnoldi iteration [48], except that no orthonormalization is incorporated between iterations. The evolution of perturbations is shown through isosurfaces of vertical velocity perturbations $\left(v^{\prime}\right)$ in Fig. 5 at nine different instants. The initial transients in the perturbation evolution (Fig. 5a) are dominated by the random nature of the initial conditions. Subsequently, however, characteristic structures exhibiting meaningful scales appear. In particular, banana-like structures [28], reminiscent of Taylor-Görtler-like (TGL) vortices as shown in Fig. 5f-i, manifest periodically at the upstream and downstream walls. The maximum number of such structures on a wall at any time is five.

These linearized snapshots are subjected to dynamic mode decomposition (DMD); the dynamic modes thus obtained are equivalent to the global linear stability modes [43]. The eigenvalue of the mode yields data on the associated frequency as well as growth rate. The lack of orthonormalization between steps in DMD is sometimes associated with convergence issues of eigenmodes compared to Arnoldi approach. Although this issue is relatively moot for dominant eigenvalues of interest, which are generally robust if a sufficiently long dataset is used $[12,41,43]$, the present results are also compared with the eigenvalues obtained using the Arnoldi approach for further validation.

Figure 6 shows the eigenspectrum obtained using this approach, with the dimensionless modal angular frequencies $(\omega)$ and growth rates $(\sigma)$ along the abscissa and ordinate, respectively. At these parameters, there are no growing modes, i.e., those with positive $\sigma$. The slowest decaying (or least stable) mode pair is observed at $\omega= \pm 0.5711$. Since the flow is in pre-bifurcation state, the stability analysis of the current basic state is consistent with the theoretical considerations. As pointed out by Sipp and Lebedev [46], prior to bifurcation, both the mean flow and the steady base flow coincide. Thus, the features obtained from the current analysis may be appropriately compared against those available from the literature at $\operatorname{Re} \lesssim \operatorname{Re}_{\mathrm{cr}}$. 


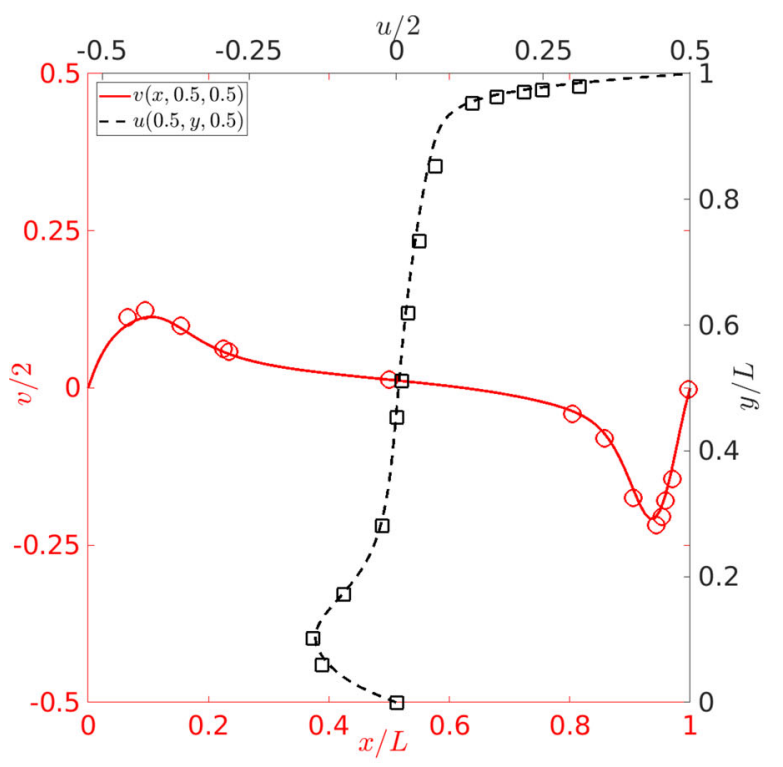

(a)

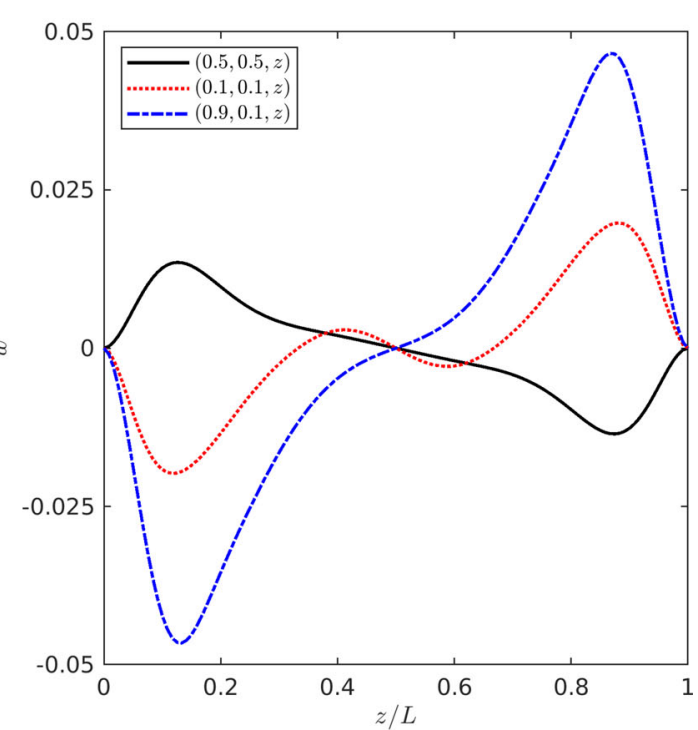

(b)

Fig. 4 a Comparison of present solution (lines) with that of Feldman and Gelfgat [11] (markers) along the centerline of the cavity at the symmetry plane. $v / 2$ and $u / 2$ velocity components plotted, respectively, along, $(x, 0.5,0.5)$ : solid red line and $(0.5, y, 0.5)$ : dashed black line. b Spanwise variation of crossflow velocity $w$ at locations - center of the cavity $(0.5,0.5, z)$, as well as near upstream and downstream secondary eddies at $(0.1,0.1, z)$ and $(0.9,0.1, z)$, respectively (color figure online)

In order to compare current results with the Arnoldi approach, the eigenvalues of dominant modes from the latter as reported in Loiseau et al. [29] are also shown in Fig. 6a. Although the reported results are at slightly higher $\operatorname{Re}(=1930)$, where an unstable mode pair is obtained, the figure presents a good comparison of dominant frequencies. As shown, the frequency of the least stable mode is very close to the reported value of $\omega= \pm 0.58$. The structure of this mode, shown in Fig. $6 \mathrm{~b}$ for $v^{\prime}$ component, is also strikingly similar to the leading eigenvector from the Arnoldi approach displayed in Fig. 6c. A similar mode shape is found in the recent high-resolution study of Gelfgat [15]. The perturbations show a mirror symmetry with respect to the symmetry plane. Figure 7a shows the eigenmodes for $u^{\prime}$ and $w^{\prime}$ velocity components. The $u^{\prime}$ mode exhibits the same symmetry as $v^{\prime}$, while $w^{\prime}$ mode shows mirror antisymmetry, consistent with the mean flow behavior. Such modes, which follow mean flow symmetry, will be referred to as 'symmetric' modes in the discussion below.

The structure of the $v^{\prime}$ mode is characterized by Taylor-Görtler-like (TGL) vortices formed along the upstream and downstream walls due to centrifugal instability. Further, at this Re, these structures are confined near the symmetry plane, since they decay away from this plane. This is the consequence of the end walls, where viscous effects attenuate the TGL structures, and the flow is strongly stabilized. This effect is absent when periodic boundary conditions are used; in this case, the TGL vortices are present throughout the domain, without modulation in the spanwise direction [50]. Thus, the critical Reynolds number for flows with periodic boundaries is smaller $\left(\operatorname{Re}_{\mathrm{cr}} \simeq 782\right)$ than when end walls are present.

The sub-dominant low-frequency modes are recovered for these flows as shown in Fig. 6a. Both the current NS-MFP approach and Arnoldi show a decaying mode at $\omega \simeq 0.17$. Loiseau et al. [29], for bifurcated flow, have suggested similar frequency to be associated with the intermittent dynamics in the flow where symmetry breaking is observed, although very briefly. We show the structure of this mode in Fig. 7b. Like the leaststable high-frequency mode $\omega_{1}$, this $\omega_{2}$ mode is also symmetric. This is consistent with the observations that the symmetry breaking is not expected at pre-bifurcation state at $\mathrm{Re}=1900$ [11]. The TGL structures for $\omega_{2}$ mode are now confined to the vicinity of only the upstream wall. A comparison of the two types of $v^{\prime}$ structures in the high- and low-frequency modes reveals that in the latter case, the TGL structures are longer, and the counter-rotating vortices observed near the upstream end of the top wall are missing. This suggests that secondary mode becomes prominent only at sufficiently high Re, i.e., when the wall jet formed at the top corner of the downstream wall has sufficient momentum to penetrate near the upstream region (see Fig. 1a). 


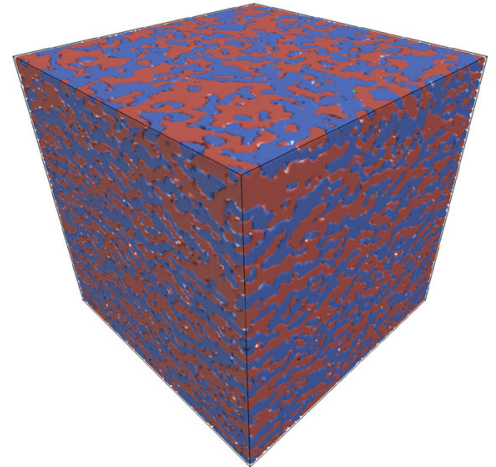

(a) $t=0.04$

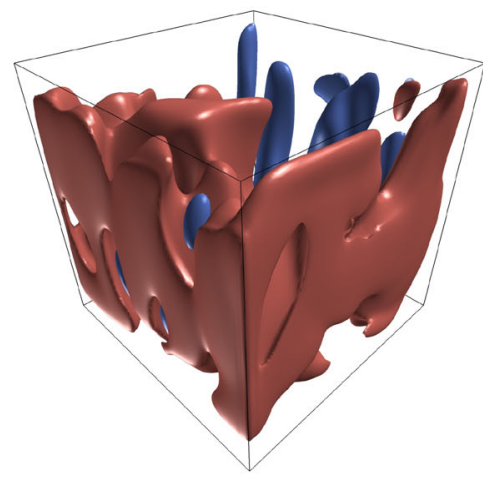

(d) $t=6$

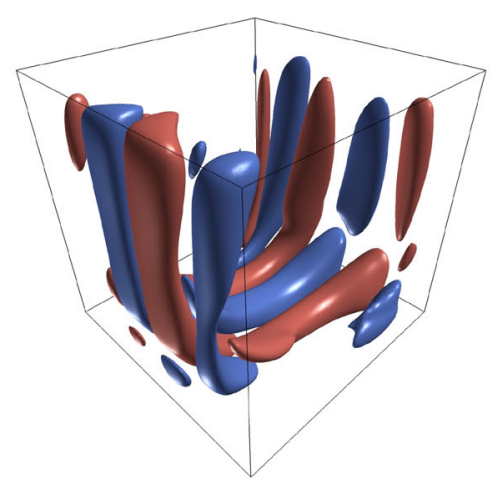

(g) $t=20$

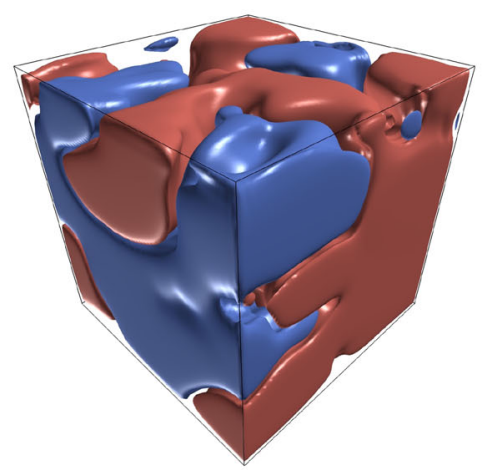

(b) $t=2$

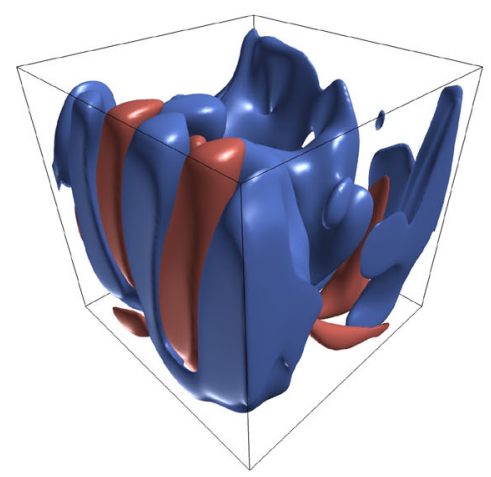

(e) $t=10$

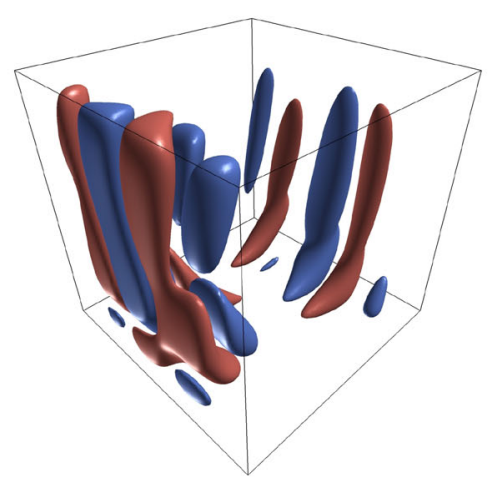

(h) $t=40$

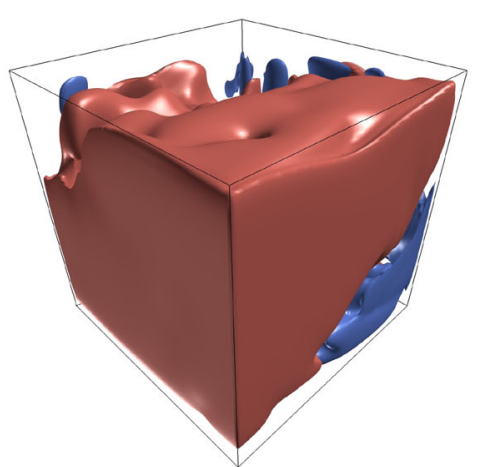

(c) $t=4$

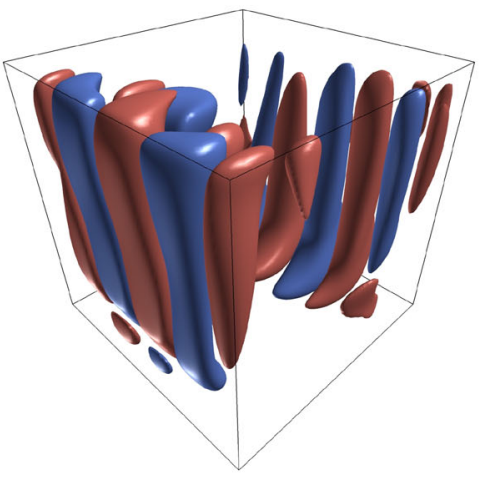

(f) $t=16$

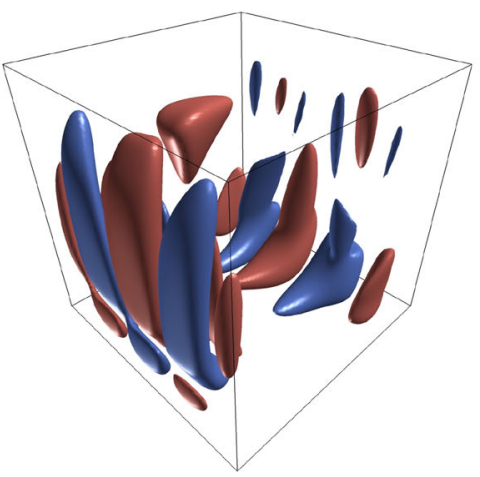

(i) $t=60$

Fig. 5 Evolution of $v$-velocity perturbations using NS-MFP $(\operatorname{Re}=1900)$

\subsection{Post-bifurcation dynamics}

Now, we examine the oscillatory and subsequent chaotic regimes for the 3D-LDC flow by considering four Reynolds numbers in $2100 \leq \operatorname{Re} \leq 3000$. The lowest Re in this range is beyond the first bifurcation point, as shown in Table 1. The change in flow character post-bifurcation is first analyzed using fully nonlinear simulations for the lowest and highest Reynolds numbers in the range. The gradual change in the flow regime due to linear dynamics is discussed subsequently.

The evolution of the nonlinear flowfield is analyzed using a velocity probe placed between the primary central eddy and upstream secondary eddy on the midplane. This is a judicious choice, since the probe point lies in the region where the instability causes strong fluctuations in velocity components [11]. The fluctuating $v$-velocity signals for $\operatorname{Re}=2100$ and 3000 are, respectively, shown in Fig. 8a, b in the top panel. Middle and 


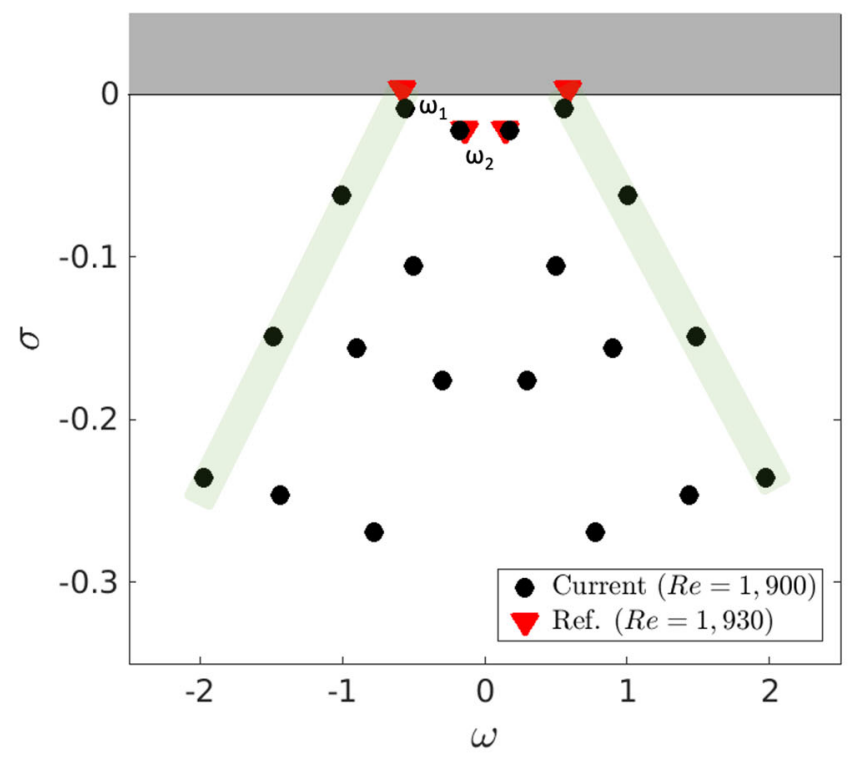

(a) Eigenspectrum

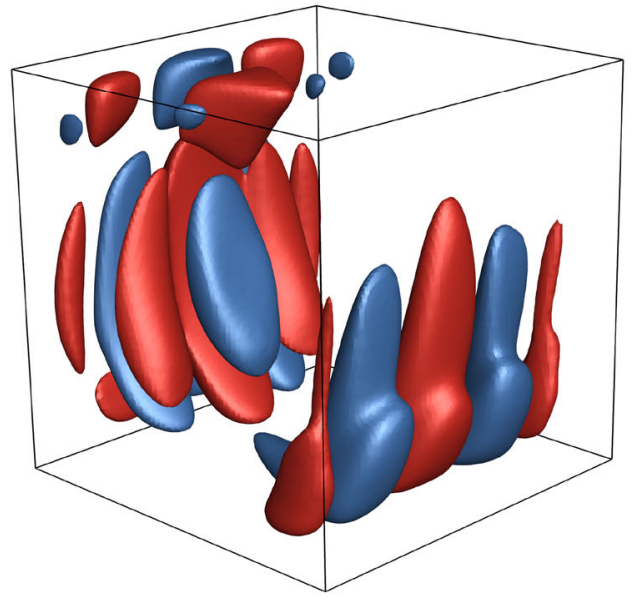

(b) Current Approach

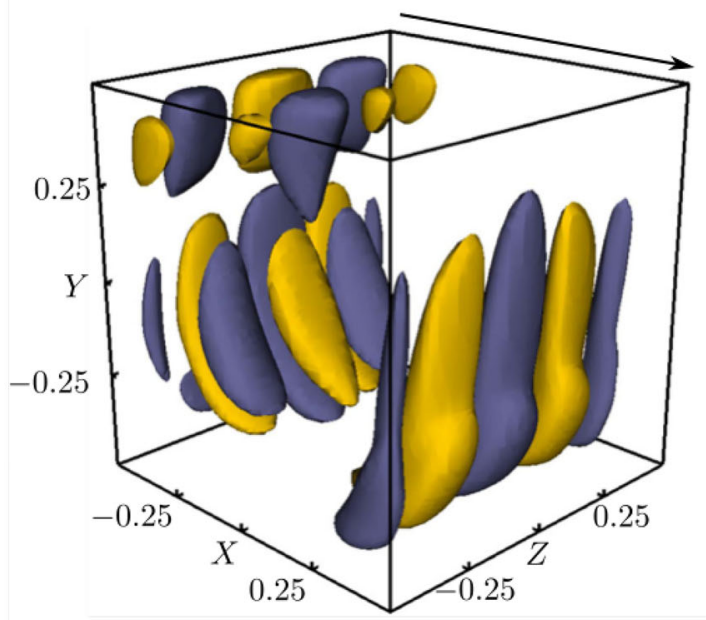

(c) Arnoldi (reproduced from Loiseau et al (2016))

Fig. 6 Eigenvalues at $\mathrm{Re}=1900$ as obtained from the current approach are shown by black circles (a). For comparison, results from Arnoldi approach at $\mathrm{Re}=1930$ as reported in Loiseau et al. [29] are also plotted in red triangles. Leading eigenmodes obtained from the current approach (b) and Arnoldi approach (c) are also shown. The green shadow box in (a) highlights the family of modes for $\omega_{1}$ consisting of the fundamental frequency and its superharmonics (color figure online)

bottom panels, respectively, display the power spectral density (PSD) estimates using the Welch approach, and wavelet transformations for these signals.

Focusing first on $\mathrm{Re}=2100$, the velocity signal in Fig. 8a clearly displays two prominent frequencies; regions where high-frequency fluctuations are apparent are marked with rectangles. The switch to highfrequency appears intermittently, soon after the flow switches from negative $v^{\prime}$ to positive $v^{\prime}$. Further, the flow asymmetry in the probed time window is evident from the fact that the maximum negative oscillation amplitude is three times bigger than that for positive fluctuations. Welch PSD estimates the two frequencies as 0.54 and 0.16 , respectively, as shown in the middle panel. These values are similar to those recovered by Lopez et al. [30] at similar flow conditions. Loiseau et al. [29] also observed these two frequencies at $\operatorname{Re}=1930$ in both linear (as discussed earlier) and nonlinear simulations. The wavelet transformation in the bottom panel indicates longer residence time of flow in the low-frequency regime at $R e=2100$, which is also reflected in 

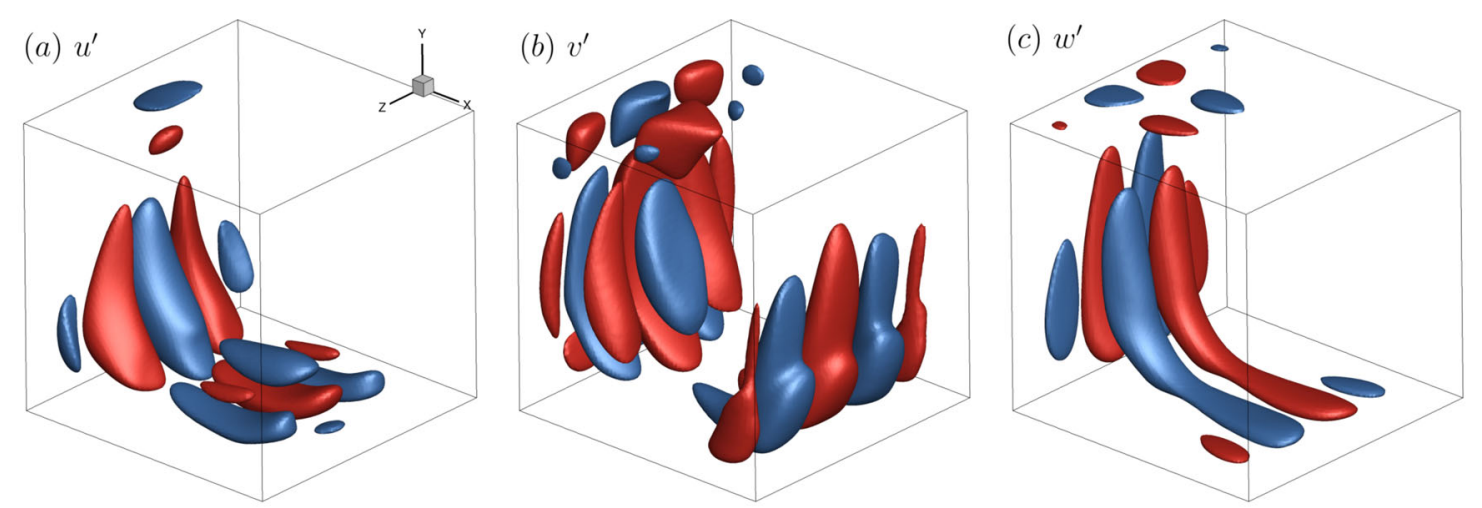

(a) $\omega_{1} \simeq 0.57$
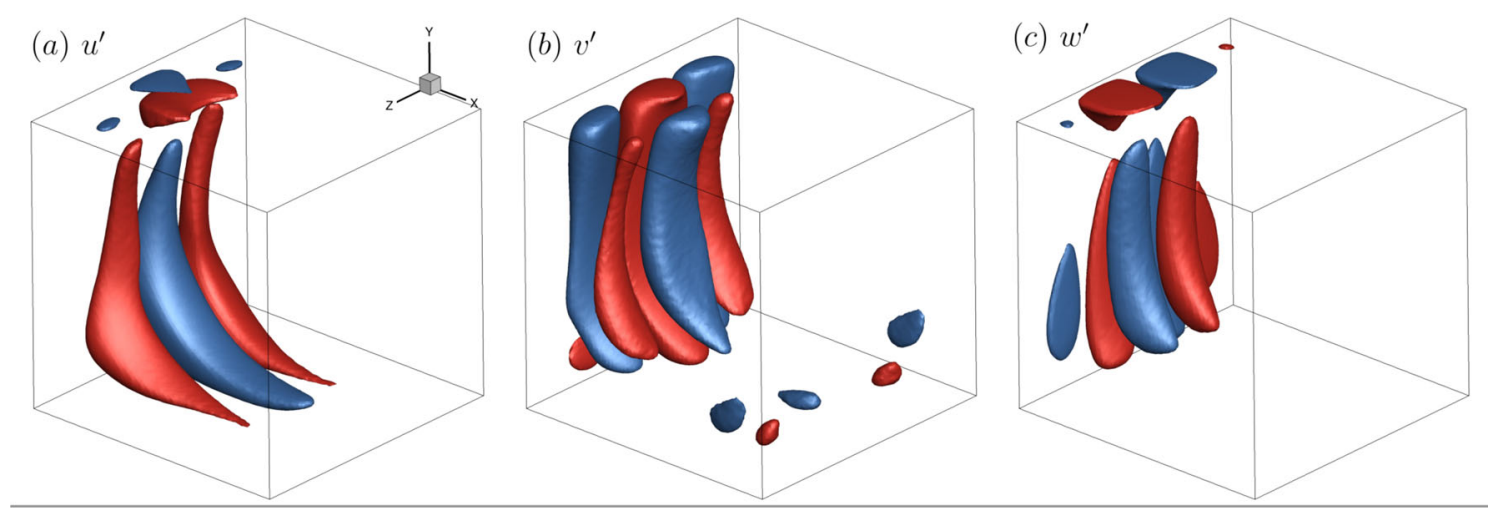

(b) $\omega_{2} \simeq 0.17$

Fig. 7 Dominant stability modes at $\operatorname{Re}=1900$. From left to right: $u^{\prime}, v^{\prime}, w^{\prime}$. Isosurfaces are plotted at $\pm 10 \%$ of the maximum values

the higher spectral energy content in the Welch estimate. The higher probability of low-frequency fluctuations in the current study is in contrast to the observations of Loiseau et al [29] at $\mathrm{Re}=1930$. This may be due to relatively higher Re used in the current study. The more dominant behavior of low-frequency events with increase in $\mathrm{Re}$ is discussed further below in the context of linear analysis.

At $\operatorname{Re}=3000$, as shown in Fig. 8b, the disparate high- and low-frequency behavior of the fluctuations is not clearly visible in the signal. High-frequency fluctuations are evident throughout with the oscillation amplitude fluctuating between -0.05 and 0.05 . This suggests a more saturated state at this Re compared to the chaotic intermittent behavior seen for $\mathrm{Re}=2100$. The PSD estimate in the middle panel shows a peak at $\omega=0.47$, but a spectral broadening observed for this state also gives considerable energy at very low frequency $\omega=0.033$. The wavelet transformation again indicates that the high-frequency content is present almost throughout the observed time window as expected from the signal.

The linear dynamics of these flows provide additional insights into the change in behavior from the stage with intermittent behavior to that with nonlinear saturation. As discussed in Sect. 1, a suitable equilibrium point for this analysis is a converged mean flow that encompasses the signature of all the limit cycles. The analysis below is therefore similar to a quasi-laminar stability analysis, as proposed in Mettot et al. [34], to extract the dominant low-frequency unsteadiness present in the flow. A natural question in the linear analysis with a timeaveraged flow is the consideration of eddy viscosity [22]. In the literature, linear analyses of mean flows have been performed using both approaches, i.e., with and without inclusion of Reynolds stress (see Mettot et al. [34]; Bhaumik et al. [6] for a list). Mettot et al. [34] have shown that even for a fully turbulent case, results from a time-averaged flow without eddy viscosity are quantitatively similar to those of the experiments, and inclusion of Reynolds stresses improves the results only slightly. For the 3D-LDC flow, Koseff and Street [24] report that the flow in a lid-driven cavity becomes turbulent at Reynolds numbers between 6000 and 8000, significantly higher than the Re-range considered here. Samantaray and Das [42] further confirmed computationally that the Reynolds stresses become significant only at $\operatorname{Re} \geq 10,000$. Therefore, as a reasonable first approximation, 

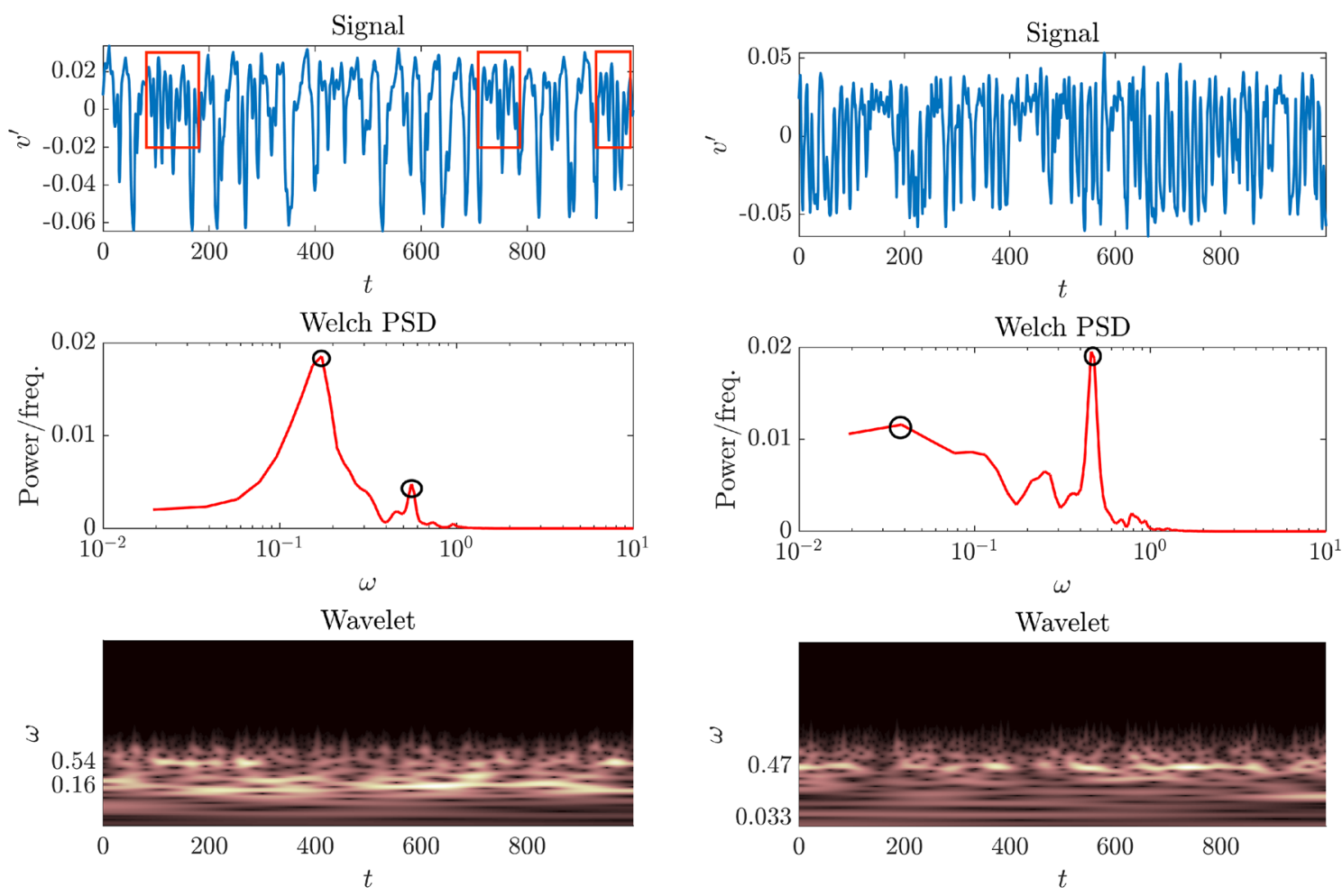

(a) $R e=2,100$

(b) $R e=3,000$

Fig. 8 Nonlinear evolution of flow at $\operatorname{Re}=2100$ (a) and 3000 (b). Top panel shows the $v$-velocity fluctuations, while Welch estimate of PSD and wavelet transformations are, respectively, shown in middle and bottom panels. Two dominant spectral peaks in both flows are marked using circle in the Welch estimate

the Reynolds stresses can be neglected in the current linear stability analyses. Subsequently, the results are compared with those inferred from fully nonlinear simulations for further verification.

The mean data are first compared with results from the literature for accuracy, and a grid convergence study is also performed. Figure 9 shows comparisons for both $\mathrm{Re}=2100$ and $\mathrm{Re}=3000$ cases at the symmetry plane, with the numerical results of Giannetti et al. [16] and experimental data of Prasad and Koseff [40], respectively. Very good agreement is evident between the current results and those from the literature for both cases. Furthermore, a grid refinement study is performed for $\mathrm{Re}=2100 \mathrm{flow}$, and the results from a fine grid $\left(201^{3}\right)$ are also plotted in Fig. 9a. Results from both meshes show good overlap, confirming grid independence of data.

The eigenspectrum obtained from the stability analysis of the flow around the $\mathrm{Re}=2100$ mean state is shown in Fig. 10. The two least stable (growing and slowest decaying) oscillatory mode pairs are shown in green and blue, respectively. The frequencies of these modes $\left(\omega_{1}= \pm 0.53\right.$ and $\left.\omega_{2}= \pm 0.11\right)$ are comparable to those found in the earlier nonlinear analysis, with a larger difference for the lower frequency. Comparing this eigenspectrum with that obtained for the stable case at $\mathrm{Re}=1900$ in Fig. 6, a primary observation is that the high-frequency, least stable modes observed at $\mathrm{Re}=1900$ have now crossed the imaginary axis and are unstable at this Re. The low-frequency modes, $\omega_{2}$, also move slightly closer to the imaginary axis, but do not cross the axis and remain stable.

The structures of these two modes, again using the $u^{\prime}, v^{\prime}$ and $w^{\prime}$ fields, are shown in Fig. 11. The mode shapes corresponding to $\omega_{1}$ are very similar in terms of their distribution as well as localization, to the TGL structures obtained for $\mathrm{Re}=1900$ (Fig. 7a). The low-frequency mode $\omega_{2}$, as shown in Fig. 7b, exhibits an antisymmetric pattern. The frequency of this limit cycle is near the observed nonlinear frequency related to intermittent symmetry-breaking events. Therefore, it may be concluded that as the Re is increased to 2100 , this limit-cycle flow assumes an antisymmetric form and manifests as the second most dominant eigenvalue in the linear analysis. 


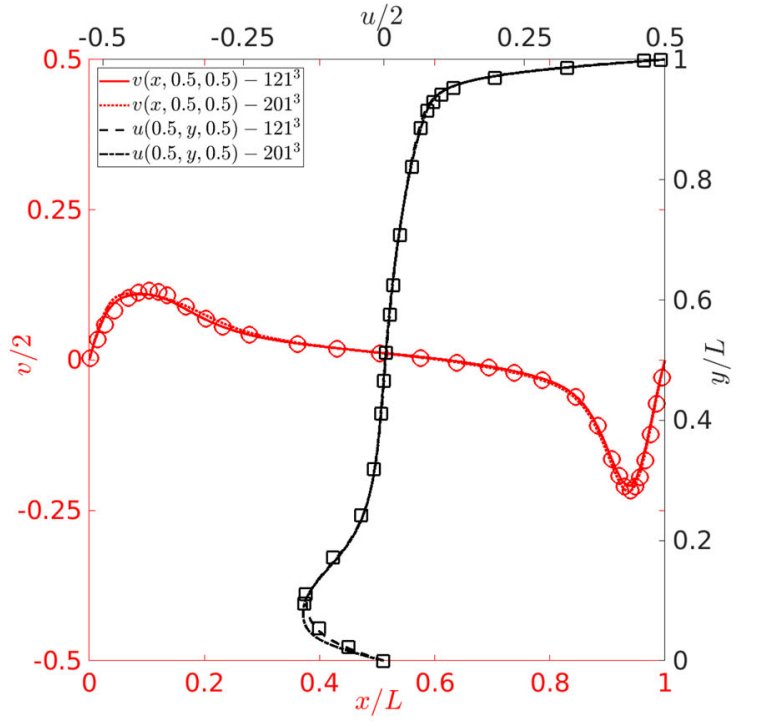

(a) $R e=2,100$

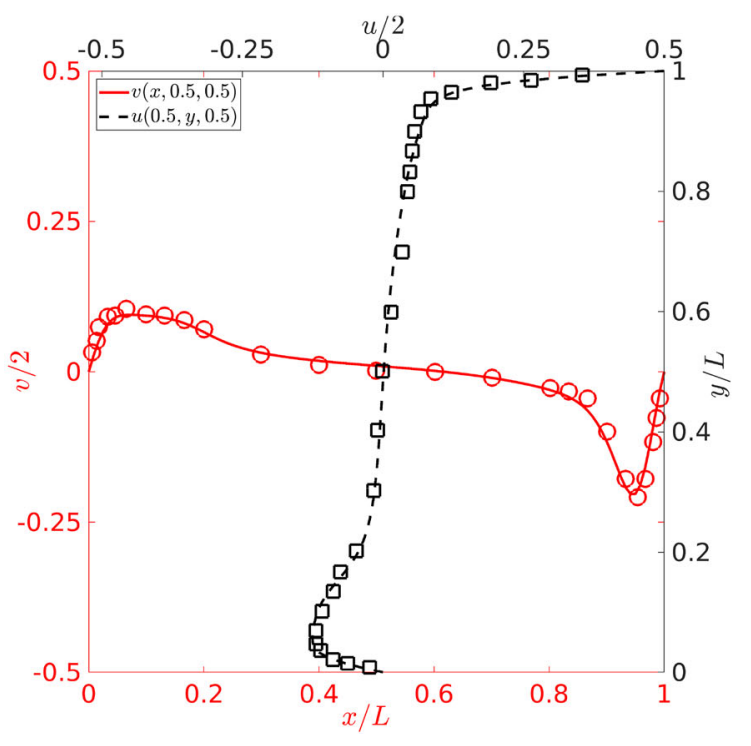

(b) $R e=3,000$

Fig. 9 Validation of mean flow at the symmetry plane. Results are compared against data from Giannetti et al. [16] at Re $=2000$ and from Prasad and Koseff [40] at $\operatorname{Re}=3200$ for $\operatorname{Re}=2100$ and 3000 cases, respectively. Grid sensitivity is shown for $\operatorname{Re}=2100$ case by performing computation on a $201^{3}$ grid

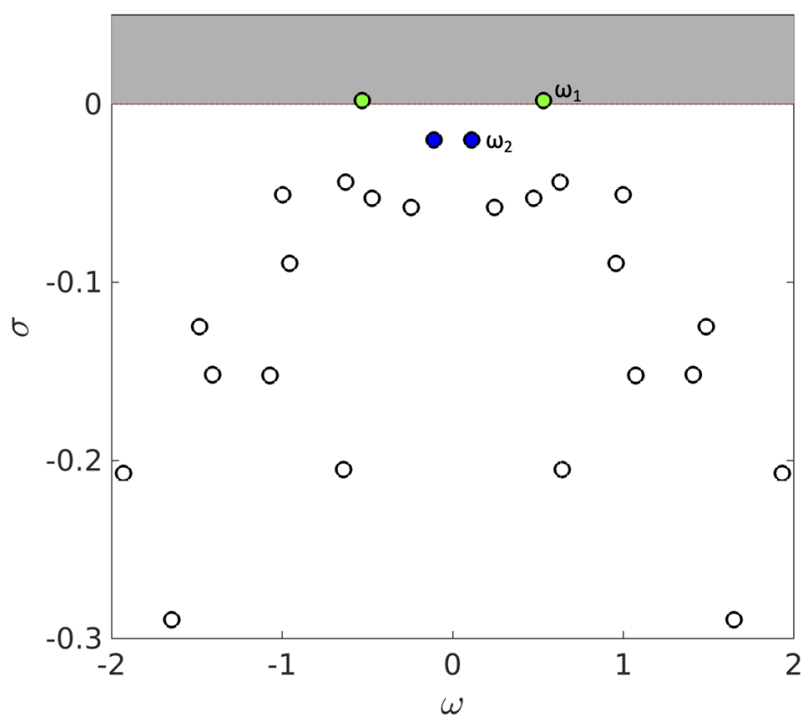

Fig. 10 Eigenspectrum at $\operatorname{Re}=2100$. Two most dominant high $\left(\omega_{1}\right)$ - and low $\left(\omega_{2}\right)$-frequency modes are highlighted in green and blue circles, respectively. Gray region shows modes which are unstable (color figure online)

The above results also suggest a hypothesis that the nonlinearly observed chaotic intermittency (also see Kuhlmann and Albensoeder [25]; Loiseau et al. [29]) is a consequence of competition between the pairs of TGL vortices at $\omega_{1}$ and $\omega_{2}$. The TGL vortices at $\omega_{1}$ result primarily due to centrifugal instability of the vortex core and hence are concentrated in the region between the primary and secondary vortices. On the other hand, vortical structures at $\omega_{2}$ indicate transverse instability in the system and assume prominence only after symmetry breaking following bifurcation.

Next, we examine the linear dynamics of the flow at the highest Re in the considered range. Figure 12 shows the eigenspectrum of the analysis at $\mathrm{Re}=3000$. The results are shown both in terms of Ritz values, as obtained from DMD analysis of the snapshots, $\lambda=\lambda_{r}+i \lambda_{i}$ (Fig. 12a, b) as well as eigenvalues $\Omega=\sigma+i \omega$ (Fig. 12c). These quantities are related by: $\Omega=\ln (\lambda) / \Delta t$, where $\Delta t$ is the time step between linearized snapshots 

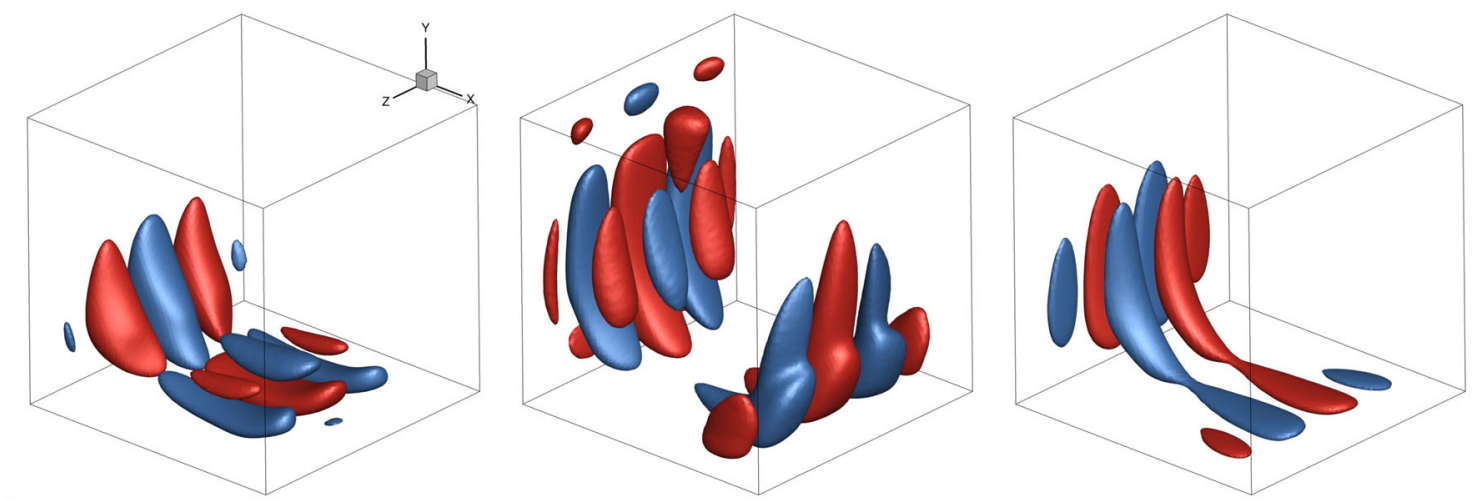

(a) $\omega_{1}$
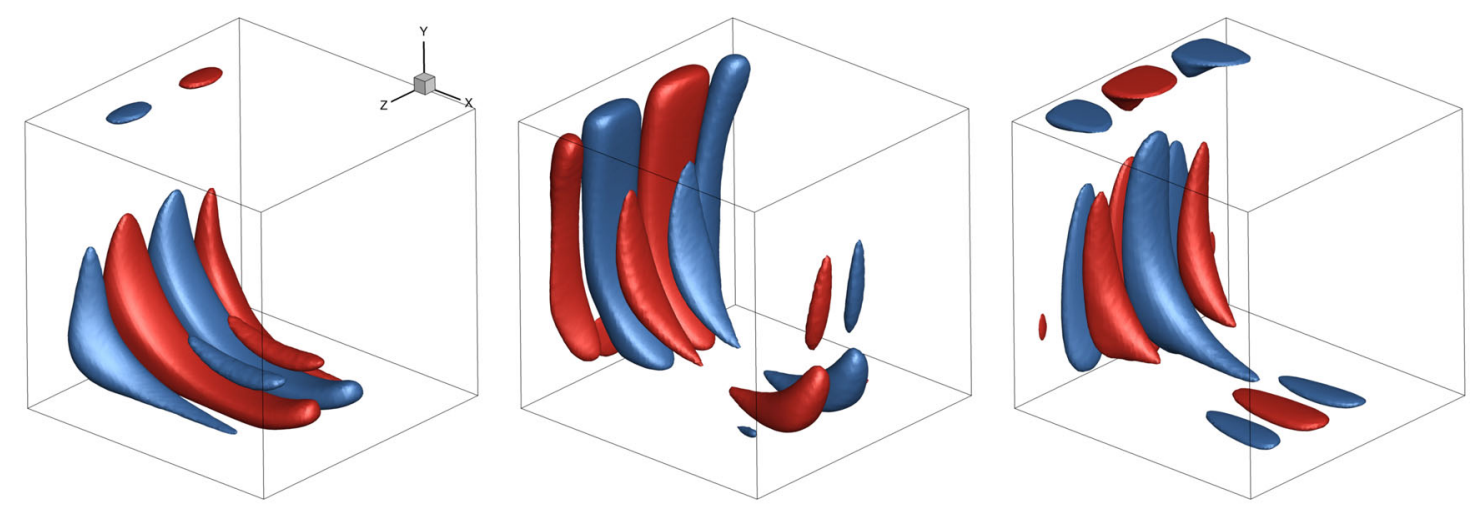

(b) $\omega_{2}$

Fig. 11 Dominant stability modes at $\operatorname{Re}=2100$. From left to right: $u^{\prime}, v^{\prime}, w^{\prime}$. Same isolevels as in Fig. 7

considered for stability analysis. The Ritz values shown in Fig. 12a lie close to the unit circle, suggesting good convergence of the results. Figure $12 \mathrm{~b}$ shows a zoomed view of the Ritz diagram near the most unstable modes, which are shown in blue and green circles. Both pairs of unstable modes lie just outside the unit circle near the $x$-axis. For a flow with saturated perturbation growth, DMD analysis with nonlinear snapshots results in all the Ritz values along the unit circle. Therefore, although the nonlinear snapshots indicate saturation as discussed in the context of Fig. 8b, linear analysis on the mean flow extracts modes as growing or decaying, reflecting the dynamics associated with large-scale structures.

The eigenspectrum obtained from these Ritz values is shown in Fig. 12c. When compared to the eigenspectrum at $\operatorname{Re}=2100$ (Fig. 10), it is evident that at $\operatorname{Re}=3000$, in addition to mode at $\omega_{1}=0.44$, the low-frequency mode $\omega_{2}=0.029$ has also become unstable. Note that in order to extract very low frequency in this case, the mean flow is obtained by averaging 1200 flow cycles after the initial transients are discarded. Further, in order to capture the low-frequency mode, the simulation is performed for 200 periods that can resolve frequencies up to 0.01 (based on the Nyquist criterion). The frequencies associated with both modes are lower compared to their respective values for $\mathrm{Re}=2100$ case, but close to the frequency peaks observed in the nonlinear analysis, as shown in Fig. 8b. Figure 12d shows the amplitudes $A$ of these modes as obtained from DMD, after optimally scaling the modes with respect to the contribution in the first snapshot, as suggested in Jovanović et al. [21]. We note that $\omega_{1}$ has the highest amplitude, followed by $\omega_{2}$. This further confirms that both unstable modes are indeed the most dominant ones in this flowfield.

The TGL structures of both these modes are shown in Fig. 13. The general form of both these modes remains consistent with those at $\operatorname{Re}=2100$, indicating that $\omega_{1}$ and $\omega_{2}$, respectively, represent symmetric and antisymmetric modes. However, a noticeable difference at high Re is that the TGL structures are no longer localized near the midplane and they now fill the previously structureless domain near the end walls. At high Re, the spanwise inhomogeneity leads to a strong crossflow which effectively extends the TGL structures to regions far from the midplane, toward the end walls. Correspondingly, the strength of TGL diminishes from the midplane to the wall. The number of dominant TGL structures for both modes, observed in $v^{\prime}$-isosurfaces in 


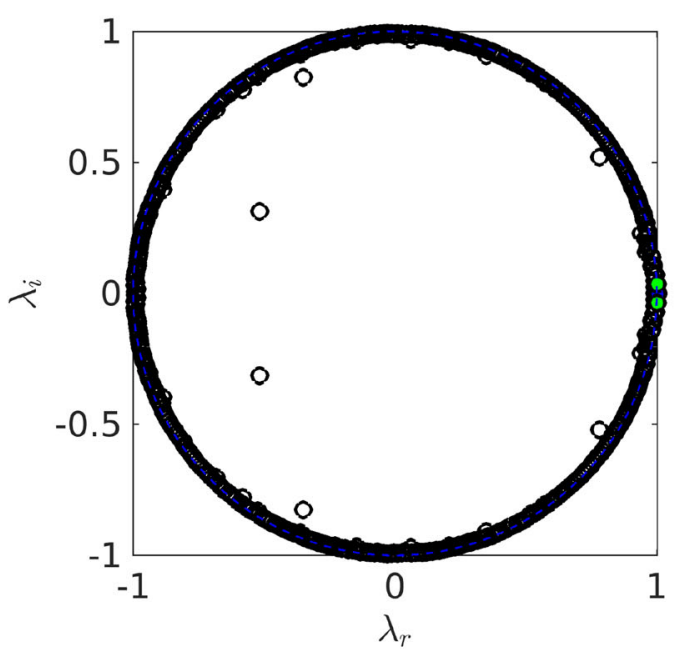

(a)

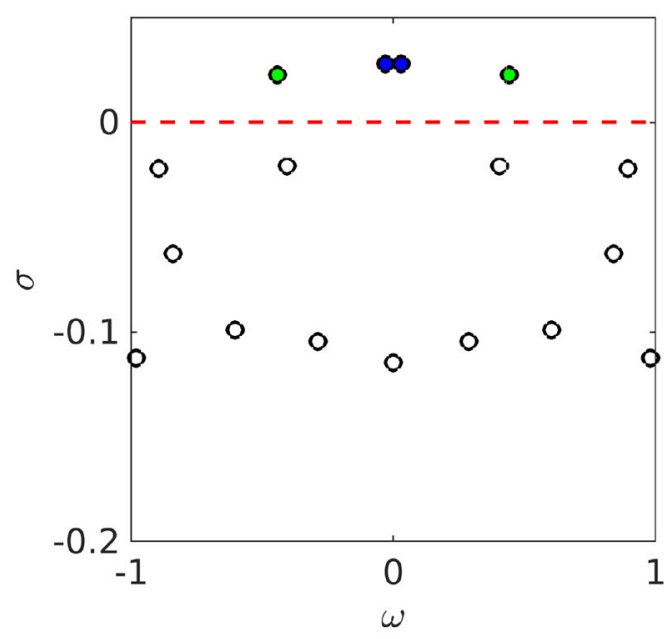

(c)

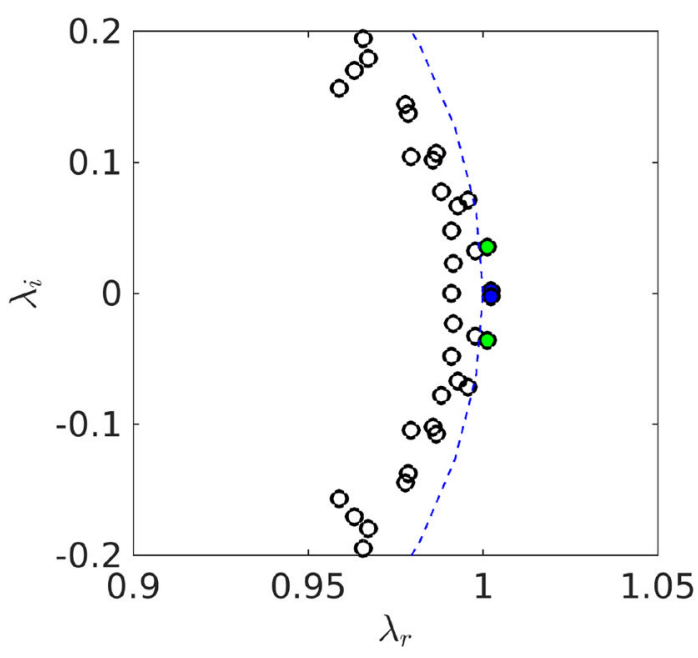

(b)

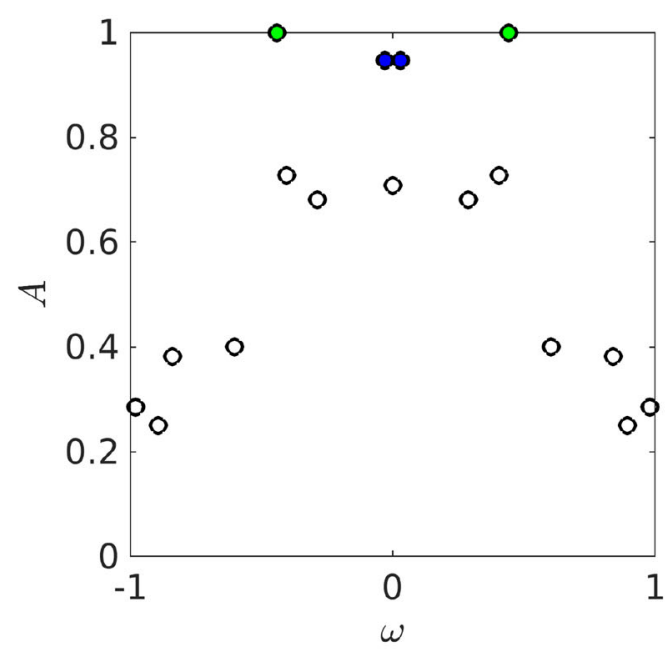

(d)

Fig. 12 Linear analysis of $\operatorname{Re}=3000$ flow. a Ritz diagram. b Zoomed view of Ritz diagram near the unstable modes. c Eigenspectrum. d Amplitude versus frequency plot

Figs. 11 and 13, increases with Re, consistent with experimental campaigns [24,44]. Figures 14 and 15 display these linear modes using $v^{\prime}$ contours on an $x z$-plane at a quarter length of the cavity from the bottom wall $(y=0.25)$. A comparison of both flows indicates that the strengths of TGL vortices increase with Re. This trend is very striking for the structures near the downstream wall, where the TGL vortices are much stronger at the higher Re. Other trends are also evident: for Re $=3000$, the TGL structures are closer to the upstream $(x=0)$ and downstream walls $(x=1)$ than the low Re case. This is associated with the larger concentration of the downward wall jets from the top right corner into a thinner boundary layer. For the mode corresponding to $\omega_{2}$, a change in wavelength of structures is also apparent.

The remarkable differences in the stability dynamics of flows at $\mathrm{Re}=2100$ and 3000 motivate a natural question about the trajectory of these modes as the flow gradually changes from one end of this Reynolds number range to the other. This question is addressed by performing linear analyses of the 3D-LDC flow at two intermediate Re, specifically 2400 and 2700, respectively. Before presenting results from this analysis, we compare the mean flow for all the cases analyzed in this study. Figure 16 shows the spanwise profiles of all three velocity components at the center of the cavity. As expected for the mean flow, $u$ and $v$ velocities are symmetric about the midplane, while $w$ is antisymmetric. As Re is increased, the peak value of all velocities decreases, with a corresponding increase in the size of central core; this is a consequence of the inability of 

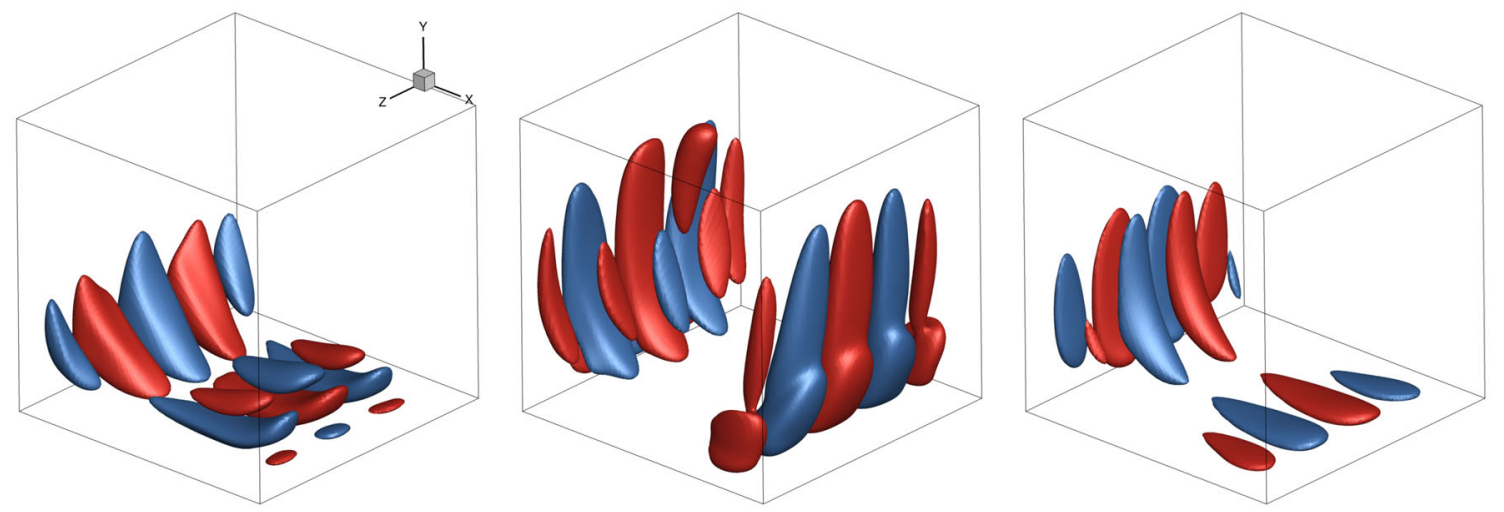

(a) $\omega_{1}$
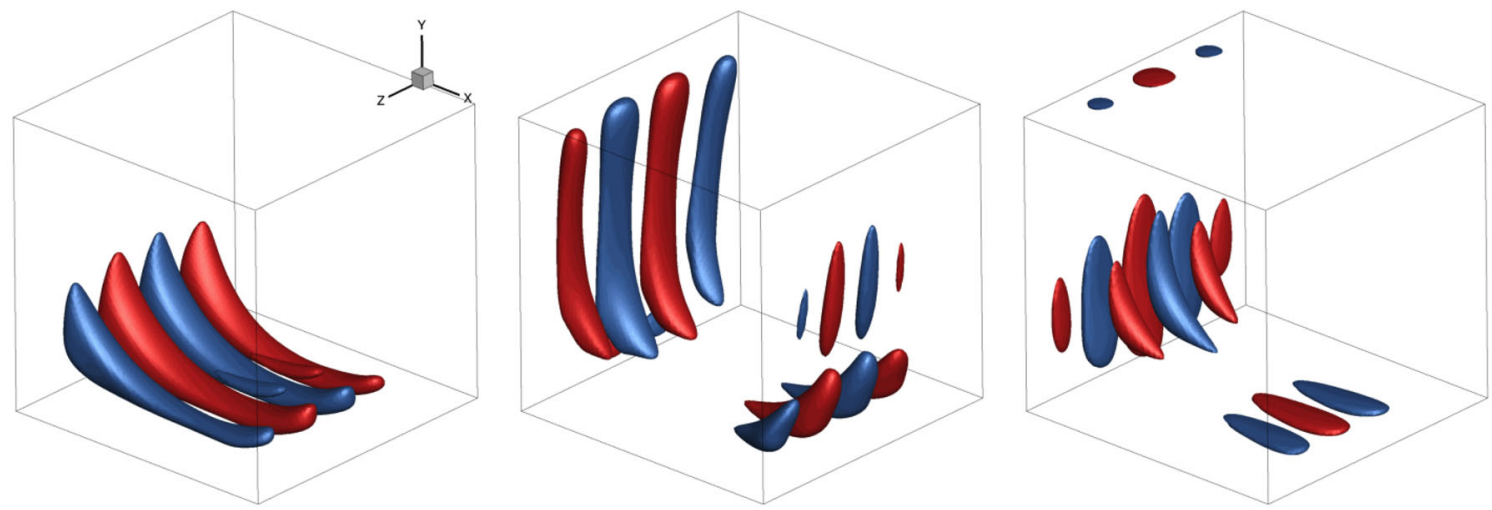

(b) $\omega_{2}$

Fig. 13 Dominant stability modes at $\mathrm{Re}=3000$. From left to right: $u^{\prime}, v^{\prime}, w^{\prime}$. Same isolevels as in Fig. 7

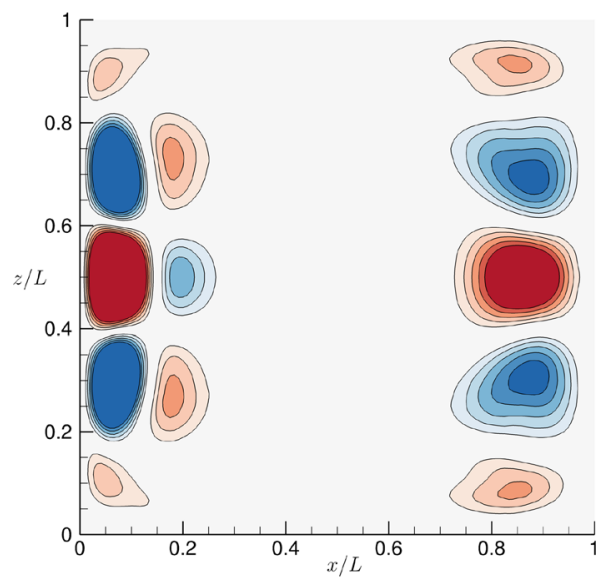

(a)। $\omega_{1}$

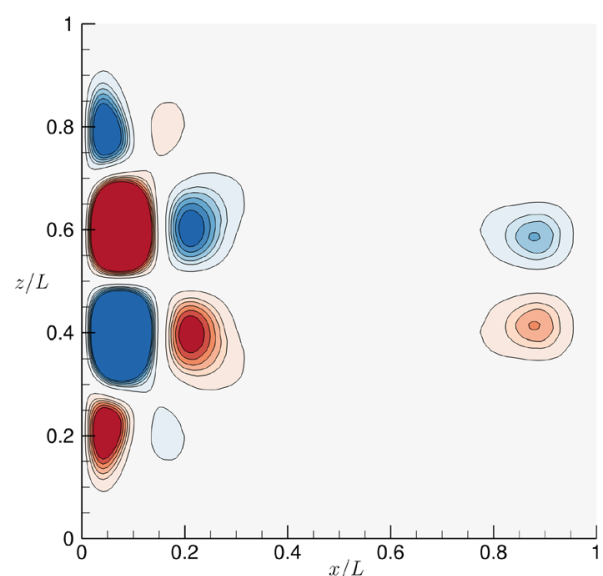

(b) $\omega_{2}$

Fig. 14 Dominant modes from linear analysis at $\operatorname{Re}=2100 . v^{\prime}$ contours at $y / L=0.25$ are shown between contour levels -0.008 and 0.008

the wall-jet energy, due to lid velocity, to penetrate into the core. The boundary layers on the end walls adjust accordingly to ensure no transverse flow in the mean sense. For the considered Re-range, very little variation is seen in the slopes of $u$-velocity boundary layer near the wall.

The results from the linear analysis of all five flows are listed in Table 2 along with nonlinear frequencies from LES for $\mathrm{Re}=2100$ and 3000 as reported earlier. Linear analysis shows a consistent drop in frequency 


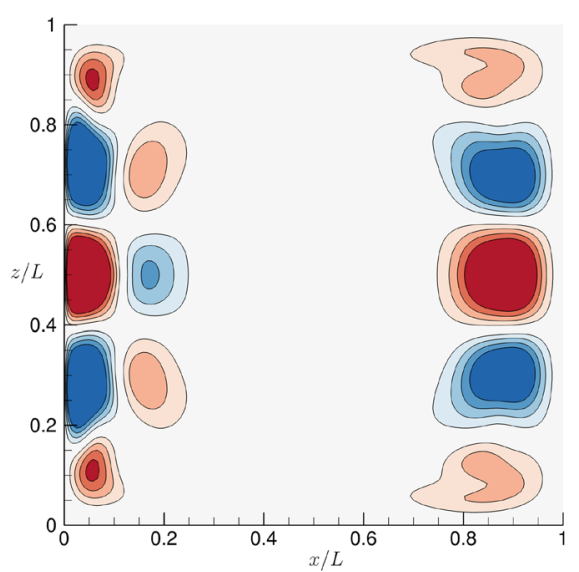

(a) $\omega_{1}$

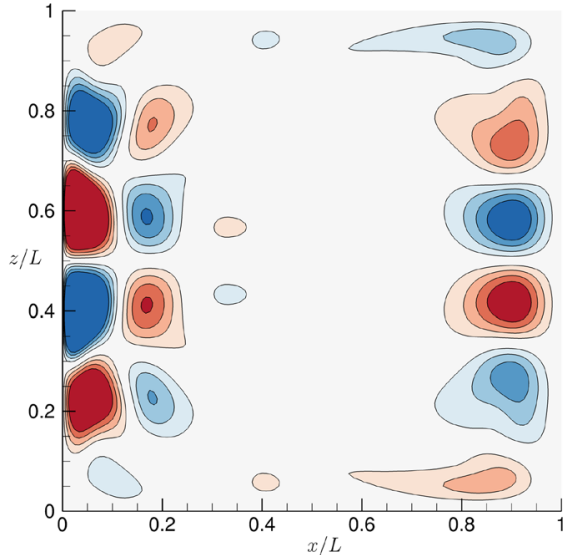

(b) $\omega_{2}$

Fig. 15 Dominant modes from linear analysis at $\operatorname{Re}=3000 . v^{\prime}$ contours at $y / L=0.25$ are shown. Same contour levels as in Fig. 14
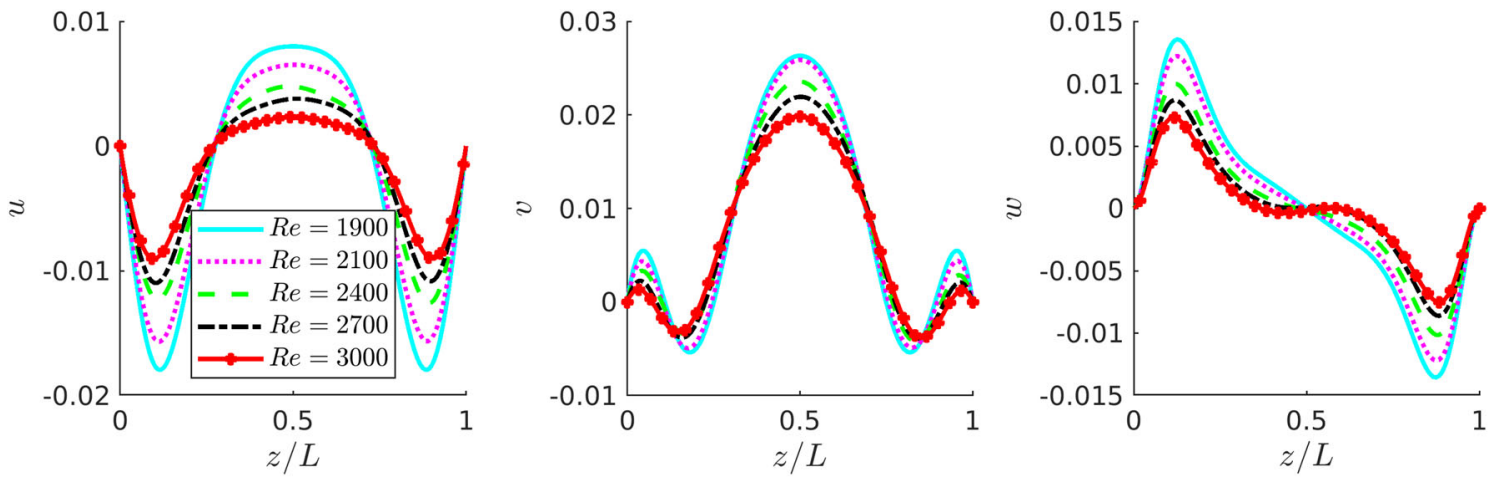

Fig. 16 Spanwise variation of mean flow at the centerline of the cavity $(0.5,0.5, z)$. Peak velocity at the center of the plane gets attenuated with increase with Re

Table 2 Frequencies and growth rates of the two most dominant linearized modes in $\operatorname{Re} \in\left[\begin{array}{lll}1900 & 3000\end{array}\right]$

\begin{tabular}{|c|c|c|c|c|c|c|}
\hline \multirow[t]{3}{*}{$\operatorname{Re}$} & \multicolumn{4}{|c|}{ Linear Analysis } & \multicolumn{2}{|l|}{ LES } \\
\hline & \multicolumn{2}{|l|}{$\omega_{1}$} & \multicolumn{2}{|l|}{$\omega_{2}$} & \multirow{2}{*}{$\frac{\omega_{1}}{ \pm \omega}$} & \multirow{2}{*}{$\begin{array}{c}\omega_{2} \\
\pm \omega\end{array}$} \\
\hline & $\pm \omega$ & $\sigma$ & $\pm \omega$ & $\sigma$ & & \\
\hline 1900 & 0.5711 & -0.0169 & 0.1736 & -0.0220 & & \\
\hline 2100 & 0.5321 & 0.0021 & 0.1108 & -0.0205 & 0.54 & 0.16 \\
\hline 2400 & 0.4942 & 0.0073 & 0.0645 & -0.0173 & & \\
\hline 2700 & 0.4696 & 0.0124 & 0.0671 & 0.0053 & & \\
\hline 3000 & 0.4424 & 0.0228 & 0.0290 & 0.0281 & 0.47 & 0.03 \\
\hline
\end{tabular}

Frequencies from two nonlinear simulations as shown in Fig. 8 are also provided

with increase in Re for both modes, while the growth rate gradually increases. In $1900 \leq R e \leq 3000$, the frequency of the most dominant mode, $\omega_{1}$, at highest Re decreases by about $23 \%$ from the value at the lowest Re, while there is a much larger reduction of about $70 \%$ for $\omega_{2}$. The very low frequency generally indicates that oscillations are not localized, but extend over a very large region in the flow, which is shown earlier in Fig. 15b. Though not shown here for brevity, the structures of modes in $2100 \leq R e \leq 3000$, corresponding to $\omega_{1}$ and $\omega_{2}$, show symmetric and antisymmetric modes, respectively. Further, as expected, with increase in Reynolds number, TGL vortices occupy a larger region in the spanwise direction along both side walls.

The eigenspectrums for all cases are plotted in Fig. 17a. Two families of modes are evident at $\omega_{1}$ and $\omega_{2}$ in all these flows; these may be associated with the two limit cycles observed in Kuhlmann and Albensoeder [25]; 


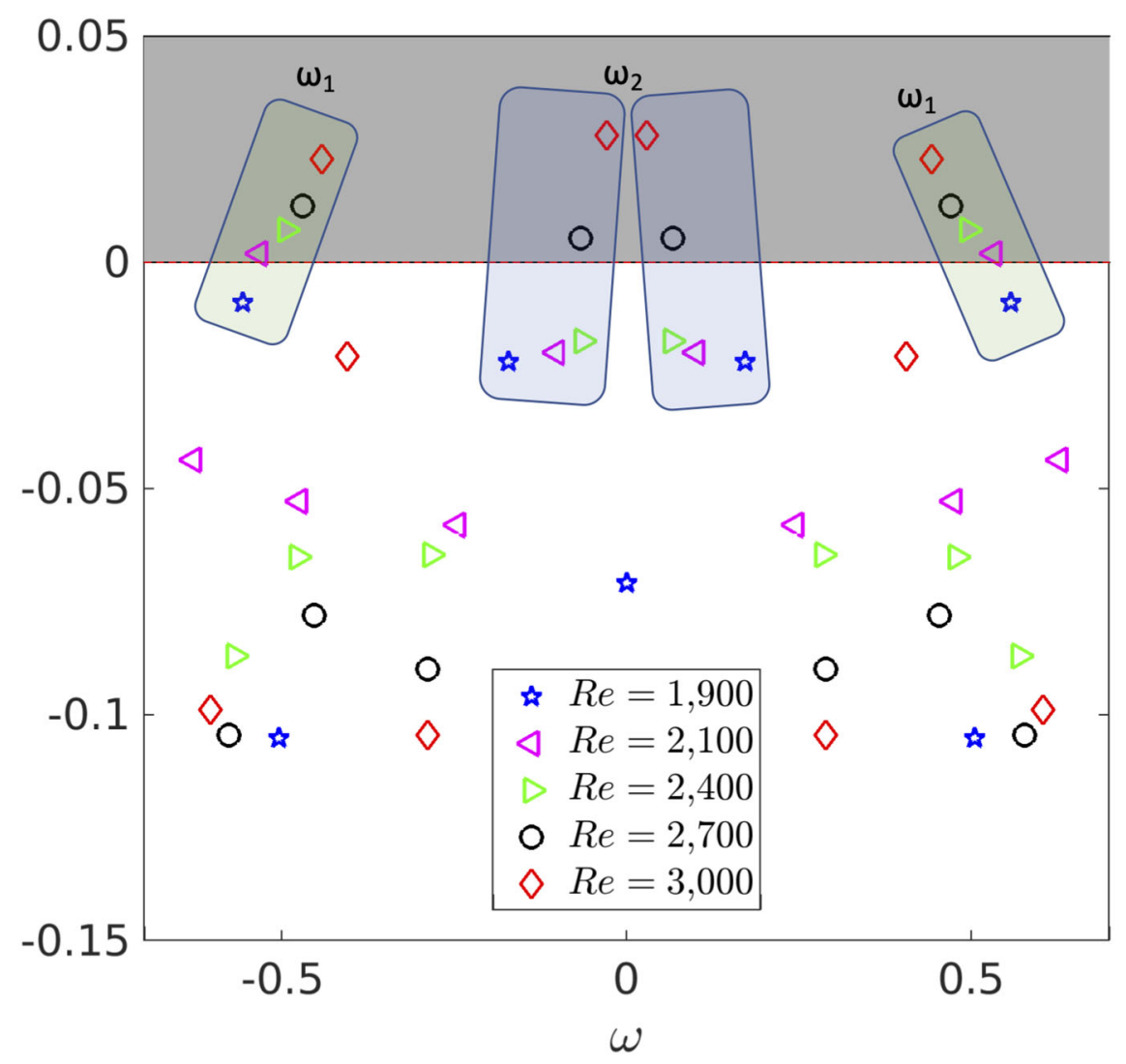

(a) Eigenspectrum

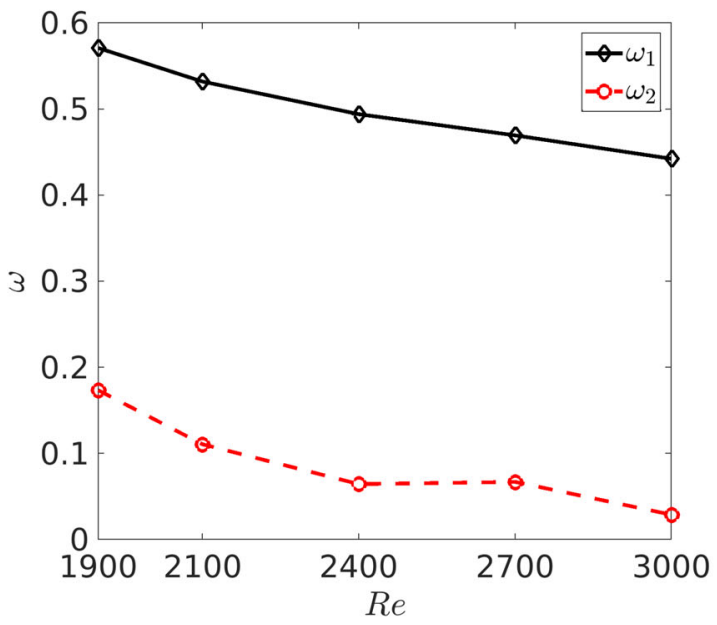

(b) Frequency vs $R e$

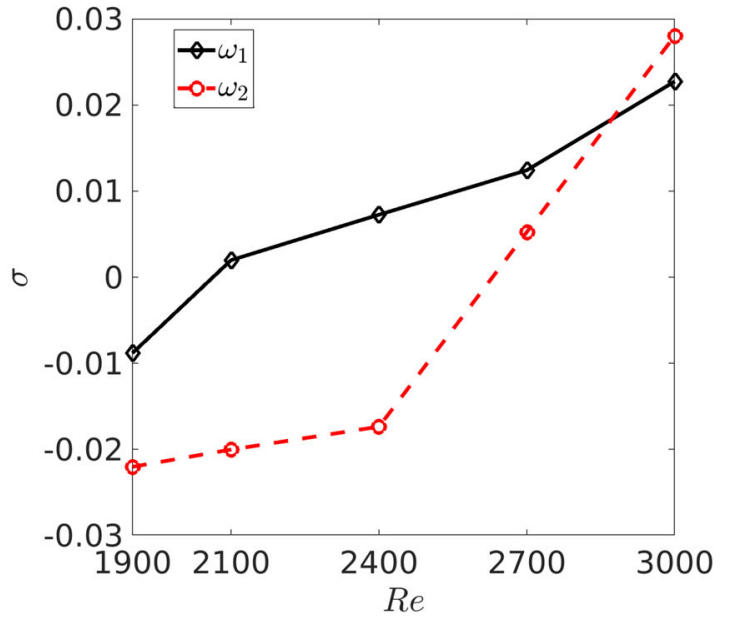

(c) Growth rate vs $R e$

Fig. 17 Stability analysis of mean flows for Re range between 1900 and 3000. Two families of modes at, $\omega_{1}$ and $\omega_{2}$, are marked

Loiseau et al. [29] and Lopez et al. [30]. While the high-frequency mode at $\omega_{1}$ is always symmetric in all cases, the low-frequency mode $\omega_{2}$ switches sign somewhere between $\operatorname{Re}=1900$ and 2100 , when the flow changes from stable to oscillatory conditions. However, the low-frequency mode remains stable until $\operatorname{Re}=2400$. At the next analysis point, $\operatorname{Re}=2700$, this mode also becomes unstable. 
Figure 17b, c shows the frequencies and growth rates of these two modes as a function of Reynolds number. The frequency decreases almost linearly with increase in Re. Examining the growth rates, the antisymmetric mode $\omega_{2}$ becomes more unstable than the high-frequency symmetric mode $\omega_{1}$ at $\operatorname{Re}=3000$, indicating that symmetry-breaking dynamics are now more dominant. The gradual increase in the growth rate of $\omega_{2}$ with Re also indicates that these results are in contrast to those of Anupindi et al. [2], who found that the symmetry is restored as the Reynolds number is increased from 2300 to 2350.

Lastly, it is reemphasized in the context of the current results that increasing Re may lead to larger changes in the mean flow, and thus the saturation due to nonlinear effects [32]; this may be especially the case at the highest Re. However, it is useful to revisit the recent observations of Bengana et al. [5] for confined flows such as in the lid-driven cavities, where the dominant dynamics are governed by geometric configurations and convection velocities. It was noted that for such flows, the nonlinear mechanism distorts the base flow only slightly (up to reasonably high Reynolds numbers), and hence, the frequencies and growth rates predicted by stability analysis around the mean flow are meaningful. Thus, when the nonlinearity becomes prominent at high Reynolds number, the primary influence is on the numerical accuracy of the observed growth rate, which is dictated by quantitative gradients in the flow. The frequencies and mode structures predicted by linear dynamics in the current study are expected to be robust [34], as also confirmed by comparisons with the nonlinear analysis.

\section{Concluding remarks}

Flows in a lid-driven cubical cavity at post-critical conditions are analyzed to provide perspective on changes in linear and nonlinear dynamics under the influence of Reynolds number. The wide Re-range considered (1900 $\leq$ $\operatorname{Re} \leq 3000$ ) encompasses flow in different regimes: from fully stable to chaotic intermittent and subsequently nonlinearly saturated as confirmed by fully nonlinear simulations. A gradual transition between the states is examined by the linear analysis. The subcritical transition of flow to oscillatory state at the bifurcation point is characterized by symmetric global modes at frequency, $\omega \simeq 0.58$. A subdominant symmetric mode at low frequency, $\omega \simeq 0.16$, is also recovered. The results are confirmed in both dynamic mode decomposition and Arnoldi iteration approaches that process the linearized snapshots. The modal spatial shapes display TaylorGörtler-like (TGL) structures, which are considered primary mechanisms governing the stability dynamics. The effects of end walls in these structures are evident in their diminished strength in the near-wall regions. Sufficiently away from the critical point, i.e., at $\mathrm{Re}=2100$, the intermittent breakup in symmetry leads to the emergence of an antisymmetric mode in the linear analysis at a much lower frequency than the primary dominant mode. However, this mode is subdominant, and the primary symmetric mode, of higher frequency $\omega \simeq 0.53$, controls the linear dynamics. These high- and low-frequency contents in the flow are also observed in the nonlinear spectral signatures. Further change in the dynamics is examined by considering three Reynolds numbers at $\operatorname{Re}=2400,2700$ and 3000. A systematic three-dimensional linear analysis at these Reynolds numbers suggests that the frequencies of the two dominant modes reduce with increase in Re. The dominance of the secondary antisymmetric mode increases as the flow moves further from the bifurcation point, indicating increased instances of symmetry breaking. Modal structures of both the primary and secondary modes show significant changes with dynamics. The TGL structures, largely confined near the midplane for $\operatorname{Re}=1900$, increasingly extend toward the end walls as the Re is increased. These changes are associated with the thinning of the boundary layer, as well as increased crossflow fluctuations with Re.

The current study adds further insights into the existing knowledge of the 3D-LDC flow, particularly in elucidating the systematic change in character with the gradual increase in nonlinearity. A considerable change in the dominant frequency of the flow during transition is an important observation that may aid in developing a control strategy in related flows. The current effort also shows the ability of a simple low-cost linear analysis tool in extracting key dynamics associated with large structures in a fully three-dimensional flow; such tools can also be exploited for more complex practical flows. Future work on this flow will examine the effects of compressibility, including leading instability dynamics and mode interaction mechanisms, to understand differences relative to the low speed counterpart studied in this paper.

Acknowledgements This material is based partly upon work supported by the Air Force Office of Scientific Research under award number FA9550-17-1-0228 (monitor: Gregg Abate). Simulations were carried out using resources provided by the US Department of Defense High Performance Computing Modernization Program and the Ohio Supercomputer Center. Several figures were made using FieldView software with licenses obtained from the Intelligent Light University Partnership Program. 


\section{References}

1. Albensoeder, S., Kuhlmann, H., Rath, H.: Three-dimensional centrifugal-flow instabilities in the lid-driven-cavity problem. Phys. Fluids 13(1), 121-135 (2001)

2. Anupindi, K., Lai, W., Frankel, S.: Characterization of oscillatory instability in lid driven cavity flows using lattice Boltzmann method. Comput. Fluids 92, 7-21 (2014)

3. Barkley, D.: Linear analysis of the cylinder wake mean flow. EPL (Europhys. Lett.) 75(5), 750 (2006)

4. Beneddine, S., Sipp, D., Arnault, A., Dandois, J., Lesshafft, L.: Conditions for validity of mean flow stability analysis. J. Fluid Mech. 798, 485-504 (2016)

5. Bengana, Y., Loiseau, J.C., Robinet, J.C., Tuckerman, L.: Bifurcation analysis and frequency prediction in shear-driven cavity flow. J. Fluid Mech. 875, 725-757 (2019)

6. Bhaumik, S., Gaitonde, D.V., Unnikrishnan, S., Sinha, A., Shen, H.: Verification and application of a mean flow perturbation method for jet noise. Aerosp. Sci. Technol. 80, 520-540 (2018)

7. Canuto, D., Taira, K.: Two-dimensional compressible viscous flow around a circular cylinder. J. Fluid Mech. 785, 349-371 (2015)

8. Crouch, J., Garbaruk, A., Magidov, D.: Predicting the onset of flow unsteadiness based on global instability. J. Comput. Phys. 224(2), 924-940 (2007)

9. Erturk, E.: Discussions on driven cavity flow. Int. J. Numer. Methods Fluids 60(3), 275-294 (2009)

10. Feldman, Y.: Theoretical analysis of three-dimensional bifurcated flow inside a diagonally lid-driven cavity. Theor. Comput. Fluid Dyn. 29(4), 245-261 (2015)

11. Feldman, Y., Gelfgat, A.Y.: Oscillatory instability of a three-dimensional lid-driven flow in a cube. Phys. Fluids 22(9), 093602 (2010). https://doi.org/10.1063/1.3487476

12. Ferrer, E., de Vicente, J., Valero, E.: Low cost 3 d global instability analysis and flow sensitivity based on dynamic mode decomposition and high-order numerical tools. Int. J. Numer. Methods Fluids 76(3), 169-184 (2014)

13. Gaitonde, D.V., Visbal, M.R.: High-order schemes for Navier-Stokes equations: algorithm and implementation into FDL3DI. Technical Report, Air Force Research Lab Wright-Patterson AFB OH Air Vehicles Directorate (1998)

14. Gelfgat, A.Y.: Visualization of three-dimensional incompressible flows by quasi-two-dimensional divergence-free projections. Comput. Fluids 97, 143-155 (2014)

15. Gelfgat, A.Y.: Linear instability of the lid-driven flow in a cubic cavity. Theor. Comput. Fluid Dyn. 33(1), 59-82 (2019)

16. Giannetti, F., Luchini, P., Marino, L.: Linear stability analysis of three-dimensional lid-driven cavity flow. In: Atti del XIX Congresso AIMETA di Meccanica Teorica e Applicata, Aras Edizioni Ancona, Italy, pp. 14-17 (2009)

17. Gómez, F., Gómez, R., Theofilis, V.: On three-dimensional global linear instability analysis of flows with standard aerodynamics codes. Aerosp. Sci. Technol. 32(1), 223-234 (2014). https://doi.org/10.1016/j.ast.2013.10.006

18. Gudmundsson, K., Colonius, T.: Instability wave models for the near-field fluctuations of turbulent jets. J. Fluid Mech. 689, 97-128 (2011)

19. Guiho, F., Alizard, F., Robinet, J.C.: Global stability analysis with compressible CFD solver. In: 43rd Fluid Dynamics Conference, American Institute of Aeronautics and Astronautics (2013). https://doi.org/10.2514/6.2013-2620

20. Iwatsu, R., Ishii, K., Kawamura, T., Kuwahara, K., Hyun, J.M.: Numerical simulation of three-dimensional flow structure in a driven cavity. Fluid Dyn. Res. 5(3), 173 (1989)

21. Jovanović, M.R., Schmid, P.J., Nichols, J.W.: Sparsity-promoting dynamic mode decomposition. Phys. Fluids 26(2), 024103 (2014)

22. Karban, U., Bugeat, B., Martini, E., Towne, A., Cavalieri, A.V.G., Lesshafft, L., Agarwal, A., Jordan, P., Colonius, T.: Ambiguity in mean-flow-based linear analysis. J. Fluid Mech. 900, R5 (2020). https://doi.org/10.1017/jfm.2020.566

23. Koseff, J.R., Street, R.: On end wall effects in a lid-driven cavity flow. J. Fluids Eng. 106(4), 385-389 (1984)

24. Koseff, J.R., Street, R.L.: Visualization studies of a shear driven three-dimensional recirculating flow. J. Fluids Eng. 106(1), 21-27 (1984). https://doi.org/10.1115/1.3242393

25. Kuhlmann, H.C., Albensoeder, S.: Stability of the steady three-dimensional lid-driven flow in a cube and the supercritical flow dynamics. Phys. Fluids 26(2), 024104 (2014)

26. Kuhlmann, H.C., Romanò, F.: The lid-driven cavity. In: Computational Modelling of Bifurcations and Instabilities in Fluid Dynamics, Springer, pp. 233-309 (2019)

27. Liberzon, A., Feldman, Y., Gelfgat, A.Y.: Experimental observation of the steady-oscillatory transition in a cubic lid-driven cavity. Phys. Fluids 23(8), 084106 (2011)

28. Loiseau, J.C.: Dynamics and global stability analysis of three-dimensional flows. Ph.D. thesis, Paris, ENSAM (2014)

29. Loiseau, J.C., Robinet, J.C., Leriche, E.: Intermittency and transition to chaos in the cubical lid-driven cavity flow. Fluid Dyn. Res. 48(6), 061421 (2016). https://doi.org/10.1088/0169-5983/48/6/061421

30. Lopez, J.M., Welfert, B.D., Wu, K., Yalim, J.: Transition to complex dynamics in the cubic lid-driven cavity. Phys. Rev. Fluids 2(7), 074401 (2017)

31. Mack, C.J., Schmid, P.J.: A preconditioned Krylov technique for global hydrodynamic stability analysis of large-scale compressible flows. J. Comput. Phys. 229(3), 541-560 (2010)

32. Mantič-Lugo, V., Arratia, C., Gallaire, F.: Self-consistent mean flow description of the nonlinear saturation of the vortex shedding in the cylinder wake. Phys. Rev. Lett. 113(8), 084501 (2014)

33. Mattingly, G., Criminale, W.: The stability of an incompressible two-dimensional wake. J. Fluid Mech. 51(2), 233-272 (1972)

34. Mettot, C., Sipp, D., Bézard, H.: Quasi-laminar stability and sensitivity analyses for turbulent flows: prediction of lowfrequency unsteadiness and passive control. Phys. Fluids 26(4), 061701 (2014)

35. Mittal, S.: Global linear stability analysis of time-averaged flows. Int. J. Numer. Methods Fluids 58(1), 111-118 (2008)

36. Oberleithner, K., Rukes, L., Soria, J.: Mean flow stability analysis of oscillating jet experiments. J. Fluid Mech. 757, 1-32 (2014) 
37. Ohmichi, Y., Suzuki, K.: Compressibility effects on the first global instability mode of the vortex formed in a regularized lid-driven cavity flow. Comput. Fluids 145, 1-7 (2017)

38. Paredes, P., Hermanns, M., Le Clainche, S., Theofilis, V.: Order $10^{4}$ speedup in global linear instability analysis using matrix formation. Comput. Methods Appl. Mech. Eng. 253, 287-304 (2013)

39. Park, J.S., Graham, M.D.: Exact coherent states and connections to turbulent dynamics in minimal channel flow. J. Fluid Mech. 782, 430-454 (2015)

40. Prasad, A.K., Koseff, J.R.: Reynolds number and end-wall effects on a lid-driven cavity flow. Phys. Fluids A 1(2), 208-218 (1989)

41. Ranjan, R., Unnikrishnan, S., Gaitonde, D.V.: A robust approach for stability analysis of complex flows using high-order Navier-Stokes solvers. J. Comput. Phys. 403, 109076 (2020). https://doi.org/10.1016/j.jcp.2019.109076

42. Samantaray, D., Das, M.K.: Nature of turbulence inside a cubical lid-driven cavity: effect of Reynolds number. Int. J. Heat Fluid Flow 80, 108498 (2019)

43. Schmid, P.J.: Dynamic mode decomposition of numerical and experimental data. J. Fluid Mech. 656, 5-28 (2010)

44. Shankar, P., Deshpande, M.: Fluid mechanics in the driven cavity. Annu. Rev. Fluid Mech. 32(1), 93-136 (2000)

45. Shu, C.W., Osher, S.: Efficient implementation of essentially non-oscillatory shock-capturing schemes. J. Comput. Phys. 77(2), 439-471 (1988)

46. Sipp, D., Lebedev, A.: Global stability of base and mean flows: a general approach and its applications to cylinder and open cavity flows. J. Fluid Mech. 593, 333-358 (2007)

47. Sipp, D., Marquet, O., Meliga, P., Barbagallo, A.: Dynamics and control of global instabilities in open-flows: a linearized approach. Appl. Mech. Rev. 63(3), (2010)

48. Sorensen, D.C.: Implicitly restarted Arnoldi/Lanczos methods for large scale eigenvalue calculations. In: Parallel Numerical Algorithms. Springer, pp. 119-165 (1997)

49. Theofilis, V.: Globally unstable basic flows in open cavities. In: AIAA 6th Aeroacoustics Conference and Exhibit, p. 1965 (2000)

50. Theofilis, V., Duck, P., Owen, J.: Viscous linear stability analysis of rectangular duct and cavity flows. J. Fluid Mech. 505, 249-286 (2004)

51. Touber, E., Sandham, N.D.: Large-Eddy simulation of low-frequency unsteadiness in a turbulent shock-induced separation bubble. Theor. Comput. Fluid Dyn. 23(2), 79-107 (2009)

Publisher's Note Springer Nature remains neutral with regard to jurisdictional claims in published maps and institutional affiliations. 